\title{
Mesure des paramètres de transport de l'eau et des substances en solution en zone saturée
}

\author{
PAR
}

J. Molinari

Ingénieur au Commissariat

it l'Energie Atomique $(\%)$
ET

\author{
J. Rochon \\ Collaborateur Temporaire de Thèse \\ au Commissariat a l'Energie Atomique (*)
}

\section{Introduction}

Les problèmes des ressources en eau et de pollution ont suscité un essor considérable des études de transferts d'eau ou de migrations conjointes d'eau et de solutés en milieu poreux.

Les mécanismes de transfert de substances en solution dans les aquifères peuvent être analysés en termes:

a) hydrodynamiques (paramètres dits "convectifs" et dispersifs exprimant la diffusion moléculaire, la dispersion cinématique, les effets résultant des distributions de vitesses dans les canalicules et chenaux, etc.)

b) physicochimiques (paramètres de sorption caractérisant les échanges au sein du fluide ou du milieu poreux, l'adsorption, la chimisorption, etc.)

Ce type d'étude doit déboucher sur l'évaluation de ces paramètres, pour n'importe quel site et n'importe quelle substance polluante, à partir :

a) d'un ou plusieurs essais de terrain (selon l'hétérogénéité supputée du site) à réaliser sur dipôle (s) représentatif (s) fournissant, par interprétation de la distribution des temps de séjour (D.T.S.) d'un traceur de l'eau entre ce (ou ces) couple (s) de forages, les paramètres hydrodynamiques d'écoulement.

b) d'essais de laboratoire, sur un ou plusieurs échantillons représentatifs de ce même terrain, fournissant les paramètres de sorption de la (ou des) substance (s) polluante (s) dont on étudie la migration.

L'obtention de ces paramètres est justifiée par la nécessité : a) de caler, en vitesse et en dispersion, les modèles mathématiques d'écoulement filaires, bidimensionnels ou tridimen-

(*) Service d'Applications des Radioéléments et des Rayonnements Centre d'Etudes Nucléaires de Grenoble sionnels utilisés jusqu'ici pour l'étude de la propagation de nuisances

b) d'appliquer à ces modèles purement hydrodynamiques les processus de sorption de substances en solution dans le fluide vecteur, processus se traduisant par des effets de retard ou d'ablation spécifiques.

En fait, lors des essais de terrain, les traceurs dont on a évoqué dans un autre exposé les risques d'interaction, délivrent des réponses affectées à un degré plus ou moins intense par les processus physicochimiques de sorption, réponses qu'il peut être nécessaire de "décoder" pour en tirer les paramètres convectifs et dispersifs du fluide vecteur.

Avant de passer en revue les méthodes qui permettent de traiter ces problèmes complexes, nous rappellerons dans un chapitre préliminaire quelques notions générales sur la nature du milieu poreux et sur l'allure des phénomènes d'interaction.

\section{Rappels sur la nature des milieux poreux}

\subsection{LES TYPES D'EAU DANS LA ZONE SATUREE [Castany (1963), Margat-Castany (1964)].}

Un aquifère poreux peut renfermer plusieurs types d'eau susceptibles d'être classés en 2 grandes catégories

- l'eau de rétention

- l'eau gravitaire

Notons de plus, la présence d'eau de constitution entrant dans la composition chimique des minéraux.

\subsubsection{L'eau de rétention}

La molécule d'eau,par son caractère polaire qui l'assimile à un aimant permanent,est attirée et fixée par certains sites électro- 
niques de la surface des matériaux. Une certaine quantité d'eau est ainsi absorbée par la roche. C'est l'eau de rétention. Ses propriétés physiques sont différentes de celles de l'eau libre.

Elle est représentée essentiellement par 2 types d'eau :

- l'eau hygroscopique

l'eau pelliculaire

L'eau pelliculaire ne peut pas se déplacer par gravité et ne transmet pas la pression hydrostatique. C'est une eau liée qui ne peut être extraite que par centrifugation (Figure 1).

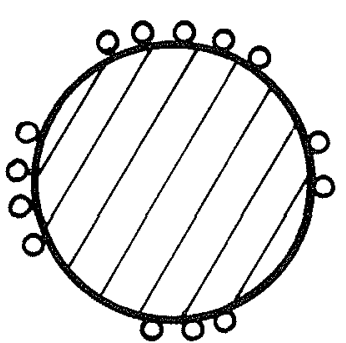

eau hygroscopique

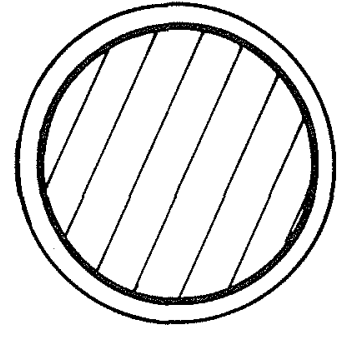

eau pelliculaire

\subsubsection{L'eau gravitaire}

Elle remplit l'espace resté libre des pores, interstices ou fissures. Cette eau de percolation (proprement dite) obéit à la force de gravité et transmet la pression hydrostatique. C'est la partie vraiment active des eaux souterraines (Figure 2).

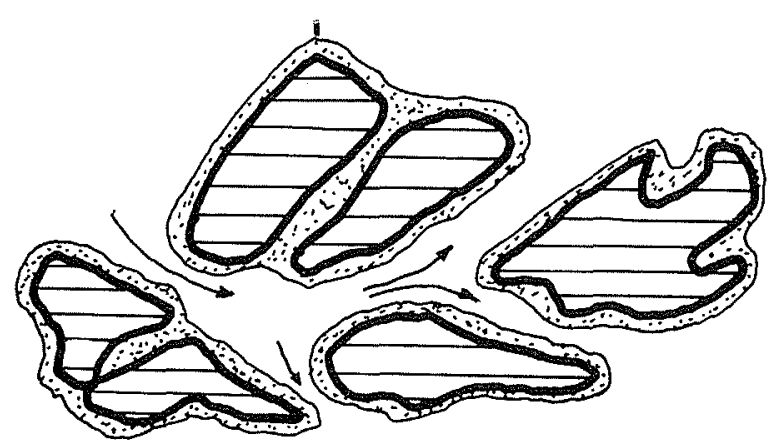
(a) $E$ (b) (c)
(b) eau de rétention
$2 /$

(a) matrice solide

\subsection{REPARTITION DES TYPES D'EAU DANS LE SOL ET LE SOUS-SOL}

Cette répartition est schématisée par les diagrammes de la figure 3 [Margat - Castany (1974)].

On notera que le pourcentage d'eau liée, est loin d'être négligeable. L'eau hygroscopique peut atteindre 15 à $20 \%$ (pour des sables fins ou moyens) de la teneur en eau. L'eau pelliculaire varie de $40 \%$ pour les argiles à $3 \%$ pour les sables. Ceci explique les différences parfois considérables entre les essais d'adsorption effectués en cuves et ceux sur colonne [Lallemand (1968)].

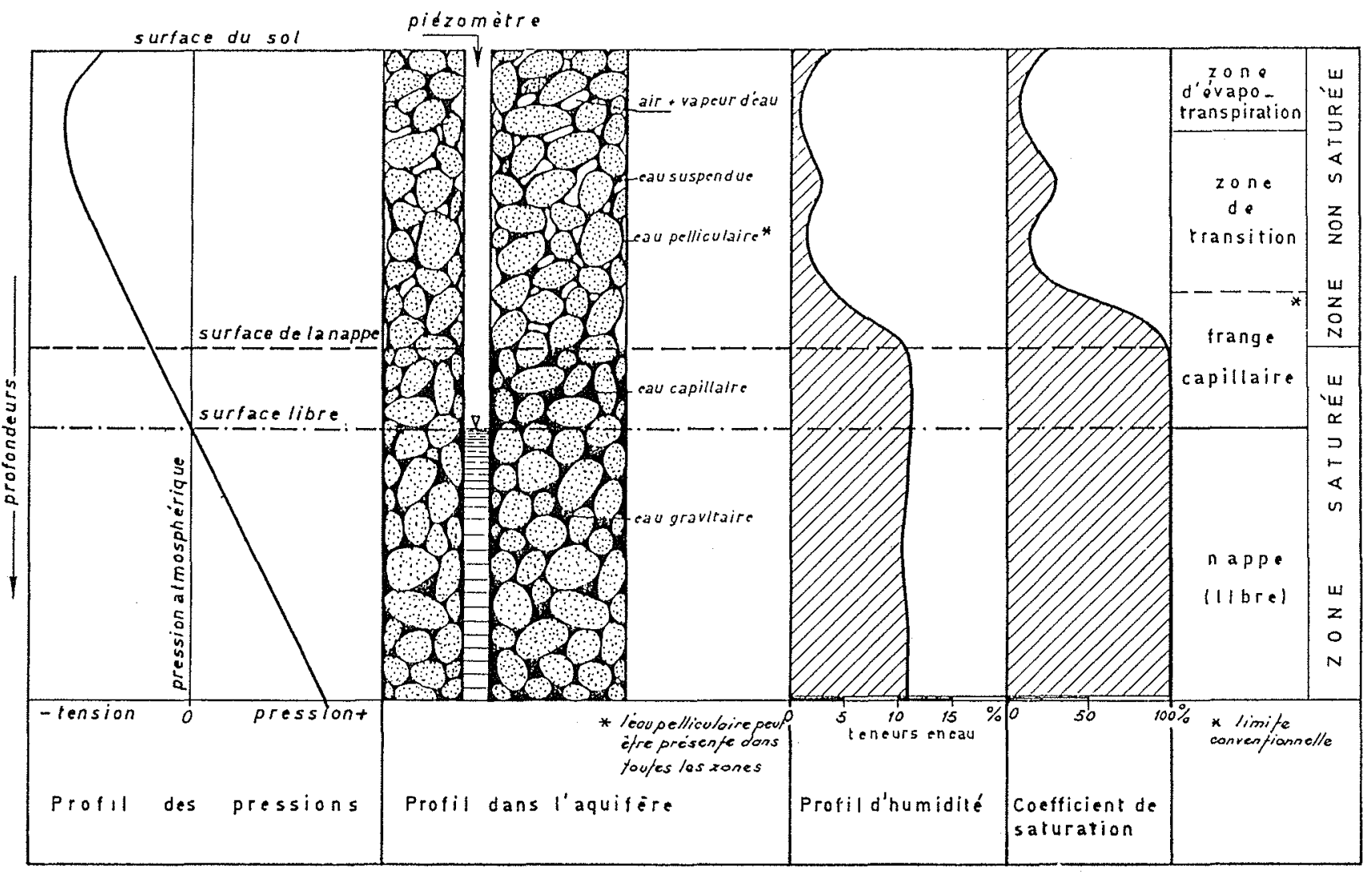

3/ Zonalité sol/eau souterraine - in Margat - Castany (1974). 


\subsection{LES PROBLEMES D'ECHELLES}

Gaudet (1974) considère que si l'on veut faire une analyse fine des phénomènes de migrations d'eau et de soluté l'on doit distinguer, en laboratoire, 3 niveaux d'approche:

- l'échelle locale

- l'échelle du volume de fluide

- l'échelle macroscopique

\subsubsection{L'échelle locale}

C'est l'échelle classique de la mécanique des fluides dans laquelle on considère le fluide comme un milieu continu. On définit à ce niveau les paramètres locaux qui décrivent une quantité physique en un point, c'est-à-dire dans un élément de volume infiniment petit, conforme aux données physiques moléculaires. Les paramètres intervenant à cette échelle sont

-... la concentration du soluté

- la masse volumique du fluide

- la viscosité

\subsubsection{L'échelle du pore}

La grandeur caractéristique est le volume d'un pore du massif poreux. Les paramètres sont définis comme des moyennes des paramètres locaux correspondants, sur un volume de fluide défini.

\subsubsection{L'échelle macroscopique}

Elle est spécifique des milieux poreux. C'est à son niveau que le mélange polyphasique peut être considéré comme un milieu continu. L'élément de volume représentatif (E.V.R.) de cette échelle est défini comme suit : autour d'un point, choisi à l'intérieur d'un pore, on construit un élément de volume fictif $\Delta U$. $\Delta U$ variant positivement à partir de zéro, on suit la variation de $\Delta V / \Delta U$ en fonction de $\Delta U, \Delta V$ étant le volume de vide de $\Delta U$.

Pour les faibles valeurs de $\Delta U$, le rapport $\Delta V / \Delta U$ varie de façon aléatoire, puis, quand $\Delta U$ augmente, ce rapport tend à devenir constant. La valeur de $\Delta U$ à partir de laquelle le rapport devient constant est l'élément de volume représentatif (Figure 4).

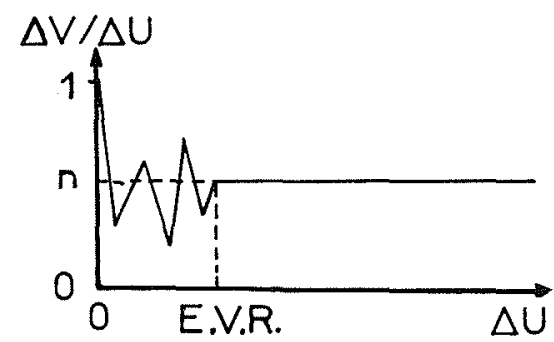

4/Elément de volume représentatif (E.V.R.) pour un milieu poreux homogène

\subsubsection{Considérations sur le passage d'un domaine à l'autre}

Le passage d'une échelle à l'autre est très délicat à exprimer. En particulier, à l'échelle locale, le milieu poreux ne peut pas être considéré comme continu et se pose, pour établir des moyennes, un problème de frontière "solide-fluide". La distribution aléatoire des pores dans le massif poreux ne facilite pas la prise en compte du problème de frontière, et tous les travaux se rapportant à ces changements d'échelles sont basés sur des hypothèses très restrictives. Aucune étude, actuellement n'est pleinement satisfaisante [Fried (1972)]
Les équations seront écrites à l'échelle de l'E.V.R. et cela nécessitera l'introduction de paramètres macroscopiques.

\subsubsection{Remarque sur la transposition des études de laboratoire aux essais de terrain [Molinari (1974)].}

La prise en considération de ces paramètres de laboratoire peut, en première approche, se révéler illusoire dans toutes les applications sur le terrain. En effet, l'hydrogéologue se refuserait à définir un E.V.R. étant données l'hétérogénéité en grand du terrain et l'existence souvent mise en évidence de cheminements préférentiels [de Cazenove (1970)] (figure 5).

\section{Rappels sur les modalités de transfert en milieu poreux}

La dispersion est l'ensemble des phénomènes mécaniques et physico-chimiques provoquant l'éloignement dans le temps et dans l'espace de particules initialement voisines.

En milieu poreux, on distingue dans les mécanismes de disper sion liés au déplacement d'une solution :

- la diffusion moléculaire

- la dispersion cinématique

- les processus de sorption-désorption

\subsection{LES MECANISMES CINEMATIQUES}

\subsubsection{Diffusion moléculaire}

C'est un phénomène physique provenant de l'agitation moléculaire. Il préexiste dans un fluide au repos. Dans le cas de solution, on peut être conduit à distinguer les processus de diffusion moléculaire de chacun des constituants de la phase liquide.

Comme nous l'avons vu en 2.1.2, l'eau, fluide vecteur, outre le fait qu'elle peut être présente dans le milieu sous la forme d'eau de constitution, est en grande partie liée au matériau (eau de rétention en milieu saturé) ou "piègée" (eau de suspension en milieu non saturé). Les molécules d'eau pénétrant dans le système sous l'effet des processus dynamiques sont susceptibles de migrer et de transiter, par diffusion moléculaire, entre ces différents domaines.

Sous l'effet de la diffusion moléculaire, le volume intéressé par le transit peut dépasser celui de la porosité efficace dans des proportions encore difficiles à préciser.

\subsubsection{Dispersion cinématique}

Quand un fluide s'écoule à travers un milieu poreux, sa distribution de vitesse n'est pas uniforme. Cela est dû :

- au profil des vitesses qui, dans les capillaires "intergrains", est parabolique, le régime laminaire ne subsistant en milieu poreux que pour des nombres de Reynolds* inférieurs à 5 [Castany (1963)]. (figure 6).

\footnotetext{
* Nombre de Reynolds :$$
R_{e}=\frac{V_{c} \cdot d \cdot \rho_{1}}{\mu}
$$

$V_{c}=$ vitesse critique moyenne $(\mathrm{cm} / \mathrm{s})$

$d=$ diamètre des pores

$\rho_{1}=$ masse spécifique du fluide $(\mathrm{g} / \mathrm{cm})$

$\mu=$ viscosité dynamique du fluide (poise).
} 
(a) - épaisseur noyée : $10 \mathrm{~m}$

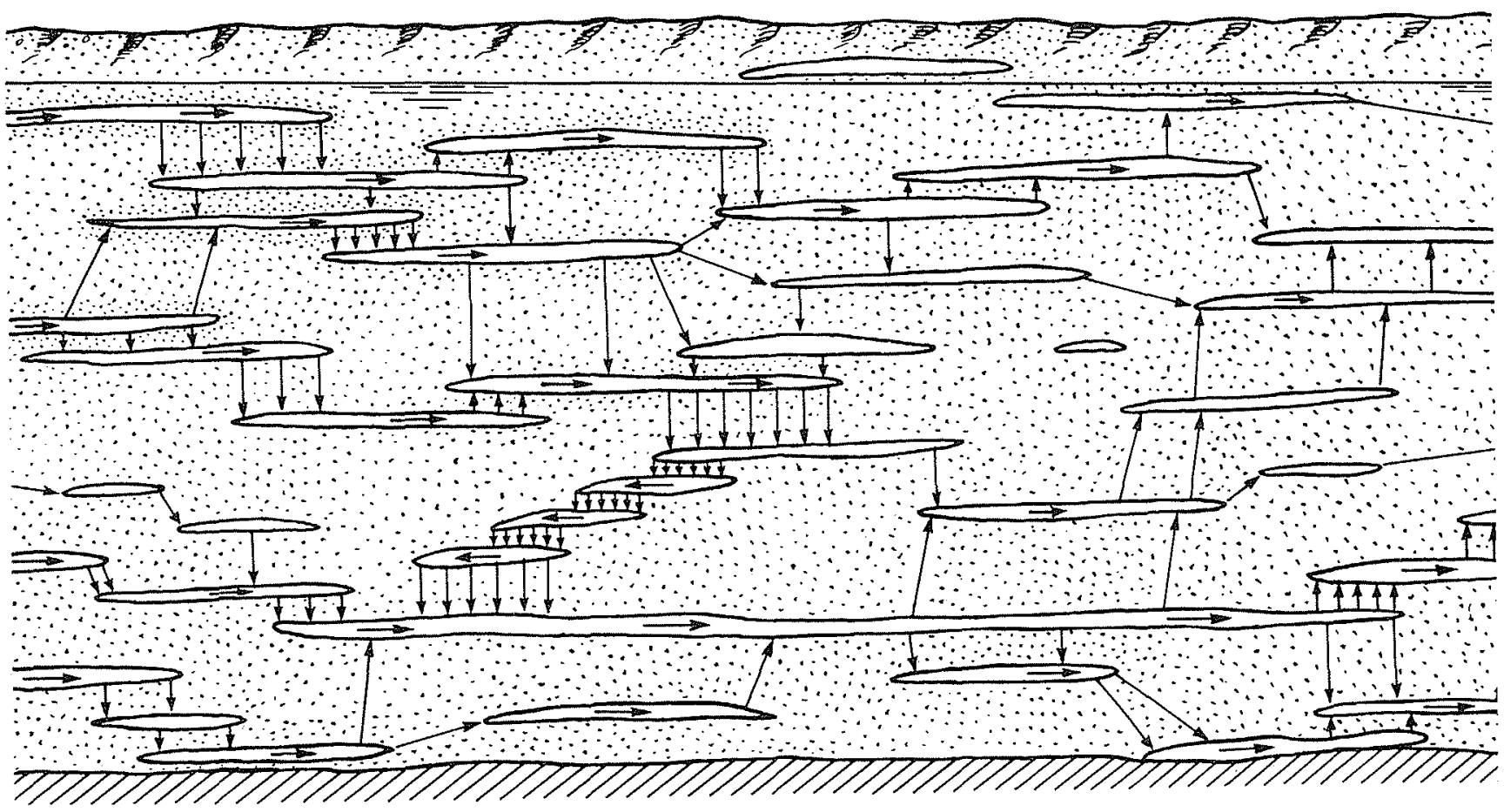

(b) - épaisseur noyée : $1 \mathrm{~m}$

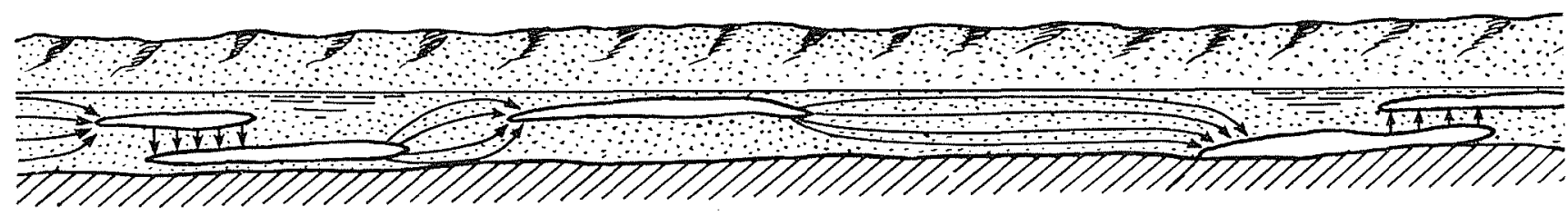

$5 /$ Lignes de courant pour un écoulement de direction moyenne horizontale dans des alluvions grossières noyées sur $10 \mathrm{~m}$ et $1 \mathrm{~m}$ d'épaisseur (échelle des hautaurs très dilatée). In De Cazenove (1970)

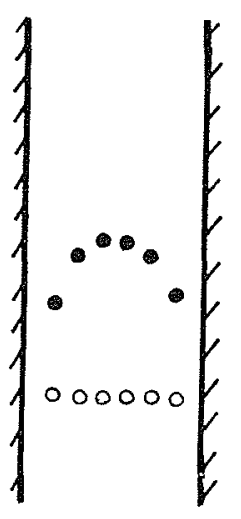

6/

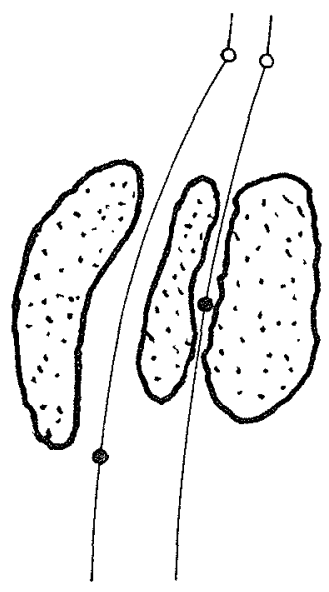

7/
- à la dimension variable des pores (figure 7).

aux lignes de courant qui fluctuent par rapport à la direction principale de l'écoulement en raison des obstacles constitués par les grains ou matériaux plus grossiers. (figure 8).
Ce n'est que dans certains cas particuliers d'hydrologie souterraine (notamment en hydrologie karstique) que la dispersion peut ètre attribuée aux turbulences d'un écoulement non laminaire. 


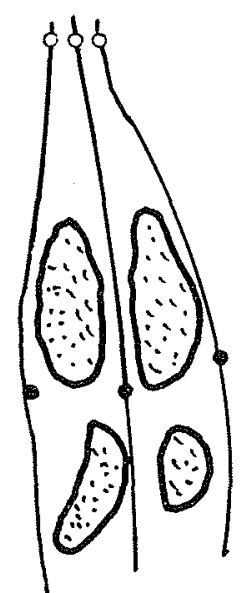

8/

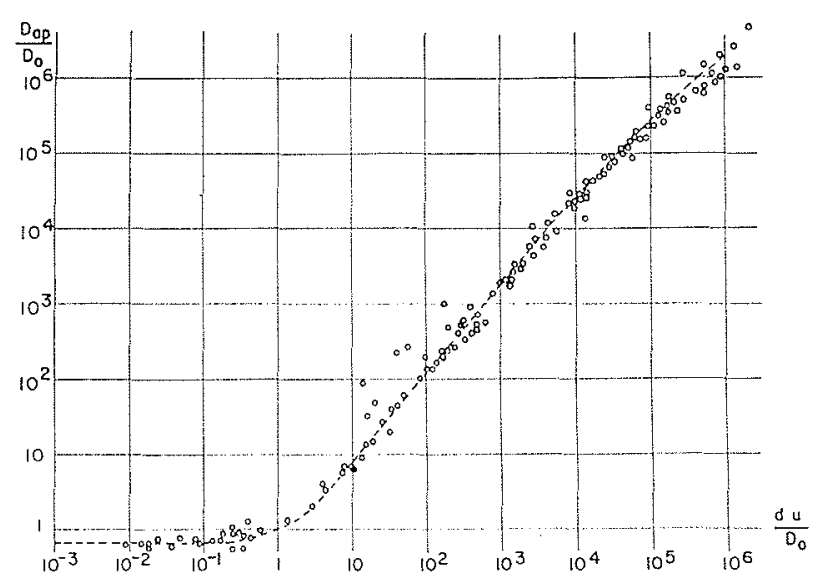

(d'OpRes PFANNKUCH 1962 in GAUDET 4974 )

$9 /$ Variation du coefficient de dispersion apparent en fonction de la vitesse moyenne de pore

l'isotherme, la facilité de désorption (isotherme de désorption). En fait, aucun de ces critères n'est satisfaisant en lui-même à cause des cas limites. Quant à l'échange d'ion, c'est une réaction ionique, donc très rapide. On le caractérisera par la capacité d'échange du solide, par la constante d'équilibre et la quantité échangée.

Les processus d'interaction sont différents suivant que l'eau de rétention est prise en compte ou non.

\subsubsection{Formes d'interfaces}

a) Interactions directes eau gravitaire (phase mobile)-solide (figure 10).

Dans cette hypothèse simplificatrice la cinétique de transfert (instantanée ou non) du soluté entre l'eau gravitaire et le solide tend à amener le système à un équilibre représenté par une isotherme. Celle-ci peut être linéaire ou non.

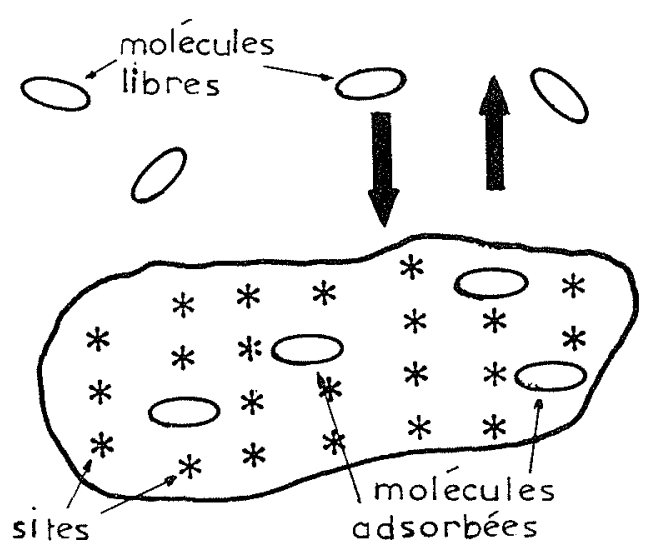

$10 /$

b) Prise en compte de l'eau de rétention (phase stationnaire). Comme nous le voyons sur la figure 11, l'eau circulant dans les cheneaux (eau gravitaire) n'entre en contact avec le matériau qu'après diffusion dans l'eau de rétention. Une telle diffusion progressant jusqu'à l'eau de constitution a été mise en évidence au moyen de traceurs intrinsèques de la molécule d'eau (eau deuteriée, eau tritiée, eau marquée à l'Oxygène 18) dont les évolutions de concentration résultent d'échanges isotopiques, cas particulier de sorption-désorption [Hanshaw (1973)]. 


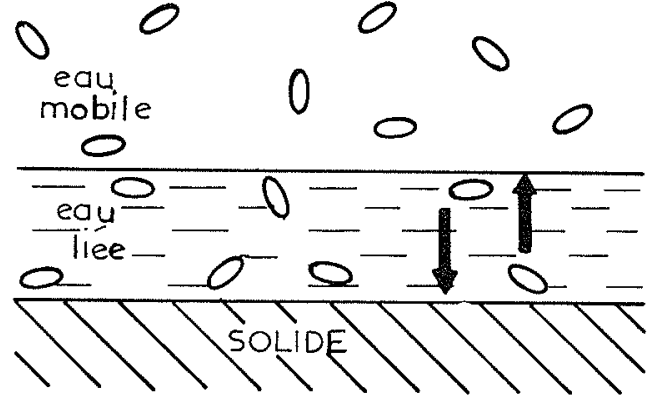

$11 /$

Tout soluté diffusera de la même façon dans l'eau de rétention avant d'atteindre la proximité du matériau où il peut être soumis à des processus de sorption. Après désorption éventuelle, c'est en sens inverse que migrera le soluté vers la solution gravitaire. L'èquilibre de sorption se fait donc entre soluté fixé et soluté dissous dans l'eau de rétention.

\subsubsection{Cinétique}

\section{a) Equilibre instantané}

Nos propres expériences et celles de nombreux autres auteurs ont montré que la cinétique de sorption de certaines substances (traceurs ou polluants) était souvent très rapide vis à vis des vitesses d'écoulement. Dans ces cas, on peut supposer que le transfert de matière est instantané et qu'on se trouve immédiatement dans les conditions d'équilibre.

\section{b) Cinétiques du ler ordre}

Lorsque la vitesse d'écoulement ne peut plus être considérée comme faible devant la vitesse de transfert, il faut considérer la cinétique du processus de sorption-désorption. La plupart des auteurs traitant de ces problèmes considèrent que la réaction est du premier ordre :c'est-à-dire que sa vitesse est proportionnelle à la concentration du soluté.

\section{c) Cinétiques d'ordre supérieur}

Elles sont rarement invoquées à cause de la complexité supplémentaire qu'elles améneraient à l'étude.

\subsubsection{Caractérisation de l'équilibre (isothermes)}

Quelle que soit la cinétique de sorption invoquée, elle tend à amener le système solide-liquide à un état d'équilibre caractérisé par une isotherme

$$
C_{s(e q)}=f\left[C_{e q}\right]
$$

où :

$$
\begin{aligned}
C_{s(e q)}= & \begin{array}{l}
\text { concentration volumique en soluté dans la phase solide à } \\
\text { l'équilibre. }
\end{array} \\
C_{e q}= & \begin{array}{c}
\text { concentration volumique en soluté dans la fraction mobile } \\
\text { ou retenue de la phase liquide à l'équilibre. }
\end{array}
\end{aligned}
$$

Les différentes formes de cette relation ont fait l'objet d'une étude bibliographique détaillée [Rochon (1974)].

La théorie de l'adsorption des liquides par les solides est moins complète que celle de l'adsorption des gaz en raison de la multiplicité des facteurs qui rendent l'étude de ce phénomène plus difficile.

Quoique la majorité des isothermes d'adsorption des solides à partir d'une solution ait l'allure initiale classique de l'isotherme de Langmuir qui postule que l'adsorption est monocouche, on invoque fréquemment la relation autrefois empirique de Freundlich qui module la relation de Langmuir.
Les formes des isothermes, très variées en fait, ont été classées par Giles (1960) (cf. figure 12).

La classification est basée sur la pente initiale et la sous classification sur sa forme à plus forte concentration.

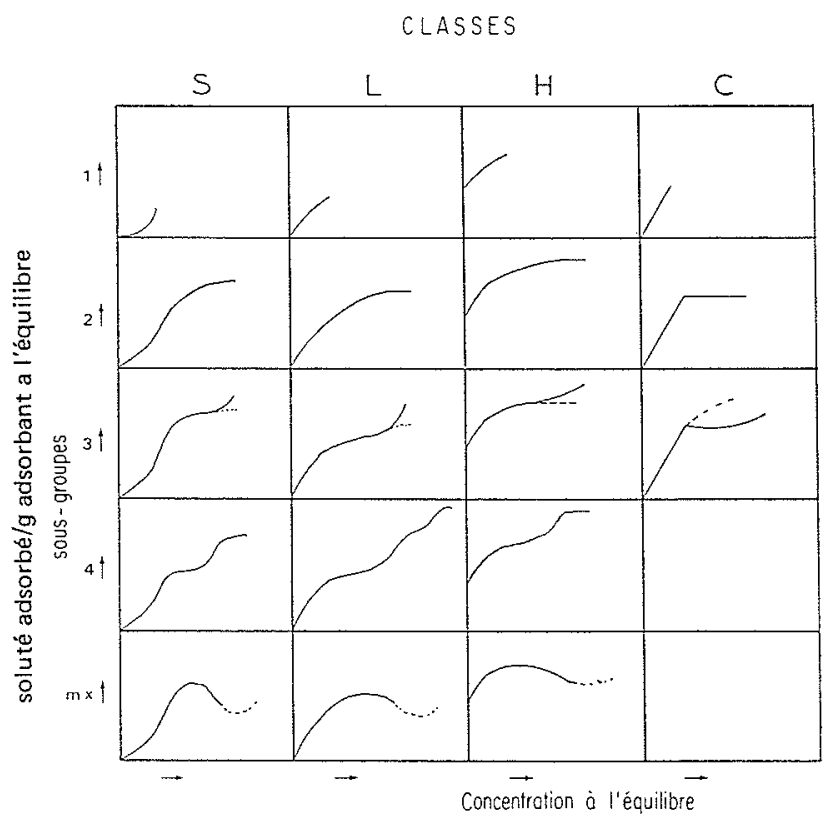

12/ Classification de Giles (11)

\section{Equation générale de la propagation unidirectionnelle de fluides miscibles dans un milieu poreux ouvert}

\subsection{HYPOTHESES SIMPLIFICATRICES}

\subsubsection{Hypothèses concernant la structure du milieu}

Les représentations les plus courantes décomposent schématiquement le milieu triphasique (gaz, liquide, solide) en au plus 4 catégories (figure 13).

- le gaz libre ou piégé (en milieu non saturé)

- le solide

- le liquide "mobile" (apparenté à l'eau gravitaire)

- le liquide "lié" ou "retenu" (apparenté à l'eau dite de rétention)

Ce milieu est considéré comme continu à l'échelle de l'élément de volume représentatif (E.V.R.).

La matrice solide est indéformable, homogène et isotrope à cette échelle.

\subsubsection{Hypothèses concernant l'écoulement}

Ces hypothèses sont les suivantes :

- l'écoulement est unidirectionnel

- l'écoulement est isotherme

- la phase gazeuse (en milieu non saturé) est à pression constante

- les variations de masse volumique et de viscosité de la solution en fonction de la concentration en soluté sont négligeables. 
MILIEUX NON SATURES
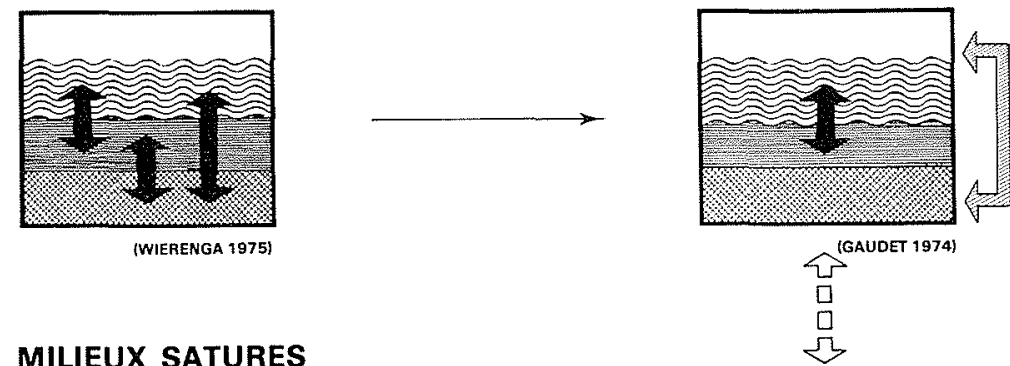

MILIEUX SATURES
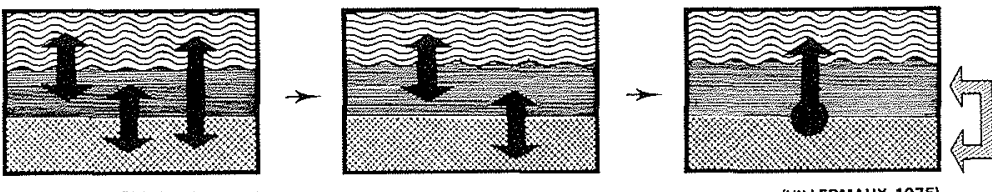

(VILLERMAUX 1975)

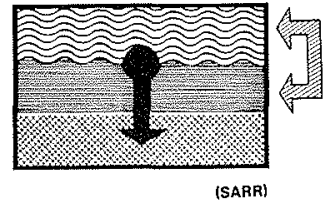

(SARR)

\section{DIFFERENTS NIVEAUX D'INTERACTION INVOQUES DANS LES} PRINCIPAUX MODELES REPRESENTATIFS DE PROPAGATION
$\bigcirc$ phase gazause
2. fraction mobile
$\Rightarrow$ fraction retenue
2olide
interactions entre phases association de phases

13/ Différents niveaux d'interaction invoqués dans les principaux modèles représentatifs de propagation

\subsection{EXPRESSION ANALYTIQUE DE LA DYNAMIQUE DE LA PROPAGATION}

Cette expression analytique est établie pour un élément de volume suffisamment petit pour considérer que toutes les grandeurs y sont uniformes et suffisamment grand pour être continu à l'échelle macroscopique (échelle de l'E.V.R.).

\subsubsection{Notations employées}

$x \quad=$ abscisse axiale

$t=$ temps

$q=$ débit unitaire de Darcy $q=\frac{Q}{\Omega}$

$Q=$ Débit volumique à travers l'élément considéré

$\Omega=$ Section de l'élément considéré

$\theta=$ Volume relatif de la solution $=\frac{\text { Volume de solution }}{\text { Volume total }}$

$\theta_{m}=$ Volume relatif de la fraction mobile

$\theta_{r}{ }^{m}=$ Volume relatif de la fraction retenue

$n=$ Porosité totale

$C_{m}=$ Concentration volumique en soluté dans la fraction mobile

$C_{r}=$ Concentration volumique en soluté dans la fraction retenue

$C^{r}=$ Concentration volumique en soluté dans toute la phase liquide : $C_{m}+C_{r}$

$C_{s}=$ Concentration volumique en soluté dans la phase solide

$D_{m}=$ Coefficient de dispersion apparent dans la fraction mobile

$D_{r}^{m}=$ Coefficient de dispersion apparent dans la fraction retenue $\left(D_{r} \simeq D_{O}\right.$ coefficient de diffusion moléculaire)

$D=$ Coefficient de dispersion apparent dans la phase liquide

$I_{m} \quad=$ Flux massique de diffusion (dans la direction d'écoulement) dans la fraction mobile

$J_{r}=$ Flux massique de diffusion (dans la direction d'écoulement) dans la fraction retenue

$k_{a}=$ constante de vitesse de sorption

$k_{d}=$ constante de vitesse de désorption
L'indice "eq" signifiera que le terme considéré est à l'équilibre. A l'exception de $\Omega$, de $n$ et de $D_{r}=D_{0}$ toutes ces quantités sont variables en fonction de l'abscisse et/ou du temps.

\subsubsection{Transport dî̀ à l'écoulement (processus convectifs)}

Ecriv ons la variation de masse entre les deux sections délimitant le volume élémentaire

Pendant un intervalle de temps $d t$, entre par la section amont

$$
\Re \tau_{1}=\Omega \cdot q(x, t) \cdot C_{m}(x, t) \cdot d t
$$

On notera que $\theta$ n'apparait pas car $q$ en tient compte implicitement (Loi de Darcy étendue au milieu poreux non saturé) Pendant ces mêmes intervalles de temps il sort par la section aval

$$
\begin{aligned}
\operatorname{RT}_{1}^{\prime} & =\Omega \cdot q(x+d x, t) \cdot C_{m}(x+d x, t) \cdot d t \\
& =\Omega \cdot\left[q(x, t)+\frac{\partial q}{\partial x} d x\right] \cdot\left[C_{m}(x, t)+\frac{\partial C_{m}}{\partial x} d x\right] \cdot d t
\end{aligned}
$$

Il s'est donc accumulé dans l'élément de volume

$$
\begin{aligned}
\pi \tau_{1}-\pi \tau_{1}^{\prime} & =-\Omega \cdot\left[q(x, t) \cdot \frac{\partial C_{m}}{\partial x}+C_{m}(x, t) \frac{\partial q}{\partial x}\right] \cdot d x \cdot d t \\
& =-\Omega \frac{\partial}{\partial x}\left[q(x, t) \cdot C_{m}(x, t)\right] \cdot d x \cdot d t
\end{aligned}
$$

4.2.3. Transport par dispersion (diffusion moléculaire et dispersion cinématique).

Comme en 4.2.2, entre dans la section amont la masse $\Re_{\zeta_{2}}=\left[\theta_{m}(x, t) \cdot J_{m}(x, t)+\theta_{r}(x, t) \cdot J_{r}(x, t)\right] . \Omega \cdot d t$ 
et il sort par la section aval

$$
\begin{array}{r}
\partial \pi_{2}^{\prime}=\left[\theta_{m}(x+d x, t) . J_{m}(x+d x, t)+\theta_{r}(x+d x, t) .\right. \\
\left.J_{r}(x+d x, t)\right] . \Omega . d t
\end{array}
$$

Il s'est donc accumulé dans l'élément de volume :

$$
\boldsymbol{N}_{2} \quad \cdots \boldsymbol{K}_{2}^{\prime}=-\Omega \cdot d x \cdot d t \cdot\left[\frac{\partial}{\partial x}\left[\theta_{m} J_{m}\right]+\frac{\partial}{\partial x}\left[\theta_{r} J_{r}\right]\right]
$$

Or, en généralisant la loi de Fick et en prenant des coefficients de dispersion apparente $D_{m}$ et $D_{r}$ dans la direction de l'écoulement, nous pouvons écrire :

$$
J_{m}=-D_{m} \frac{\partial C_{m}}{\partial x} \quad \text { et } \quad J_{r}=-D_{r} \frac{\partial C_{r}}{\partial x}
$$

d'oú :

$$
\boldsymbol{N}_{2}-\boldsymbol{r c}_{2}^{\prime}=\Omega \cdot d x \cdot d t \cdot\left[\frac{\partial}{\partial x}\left[D_{m} \theta_{m} \frac{\partial C_{m}}{\partial x}\right]+\frac{\partial}{\partial x}\left[D_{r} \theta_{r} \frac{\partial C_{r}}{\partial x}\right]\right]
$$

\subsubsection{Variation du stock de soluté (dont l'interaction avec} la matrice solide)

Il faut tenir compte des fractions mobile $(m)$, retenue $(r)$ et sorbée $(s)$.

$\mathrm{Au}$ temps $t$, la masse de soluté présente à l'intérieur est :

$$
\begin{aligned}
\pi_{3}=\Omega \cdot d x \cdot\left[\theta_{m}\left(x_{1}, t\right) \cdot C_{m}(x, t)\right. & +\theta_{r}(x, t) \cdot C_{r}(x, t) \\
& \left.+(1-n) \cdot C_{s}(x, t)\right]
\end{aligned}
$$

au temps $t+d t$ subsiste

$$
\begin{aligned}
\pi \tau_{3}^{\prime}= & \Omega \cdot d x \cdot\left[\theta_{m}(x, t+d t) \cdot C_{m}(x, t+d t)\right. \\
& \left.+\theta_{r}(x, t+d t) \cdot C_{r}(x, t+d t)+(1-n) \cdot C_{s}(x, t+d t)\right] \\
= & \Omega \cdot d x \cdot\left[C_{m} \cdot \theta_{m}+\frac{\partial}{\partial t}\left[C_{m} \cdot \theta_{m}\right] \cdot d t+C_{r} \cdot \theta_{r}\right. \\
& +\frac{\partial}{\partial t}\left[C_{r} \cdot \theta_{r}\right] \cdot d t+(1-n) \cdot\left[C_{s}+\frac{\partial C_{s}}{\partial t} \cdot d t\right]
\end{aligned}
$$

La variation entre $t$ et $t+d t$ est donc de :

$$
\begin{aligned}
\pi_{3}-\pi_{3}^{\prime}=\Omega \cdot d x . d t & {\left[\frac{\partial}{\partial t}\left[C_{m}, \theta_{m}\right]\right.} \\
+ & \left.\frac{\partial}{\partial t}\left[C_{r} \cdot \theta_{r}\right]+(1-n) \frac{\partial C_{s}}{\partial t}\right]
\end{aligned}
$$

4.2.5. Expression analytique globale (voir tableau $n^{\circ} 1$ )

a) Expression générale

Ecrivons le bilan total :

$($ transport convectif $)+($ transport par dispersion $)=($ variation du stock $)$

Après simplification nous obtenons l'expression analytique recherchée :

\begin{tabular}{|c|c|}
\hline $\begin{array}{c}\frac{\partial}{\partial x}\left[q C_{m}\right]+\frac{\partial}{\partial x}\left[D_{m} \theta_{m} \frac{\partial C_{m}}{\partial x}\right]+\frac{\partial}{\partial x}\left[D_{r} \theta_{r} \frac{\partial C_{r}}{\partial x}\right] \\
\text { Convection }\end{array}$ \\
$=\frac{\partial}{\partial t}\left[C_{m} \theta_{m}\right]+\frac{\partial}{\partial t}\left[C_{r} \theta_{r}\right]+(1-n) \frac{\partial C_{s}}{\partial t}$ \\
Variation du stock
\end{tabular}

Tableau 1

Expressions analytiques de propagation en milieu poreux.

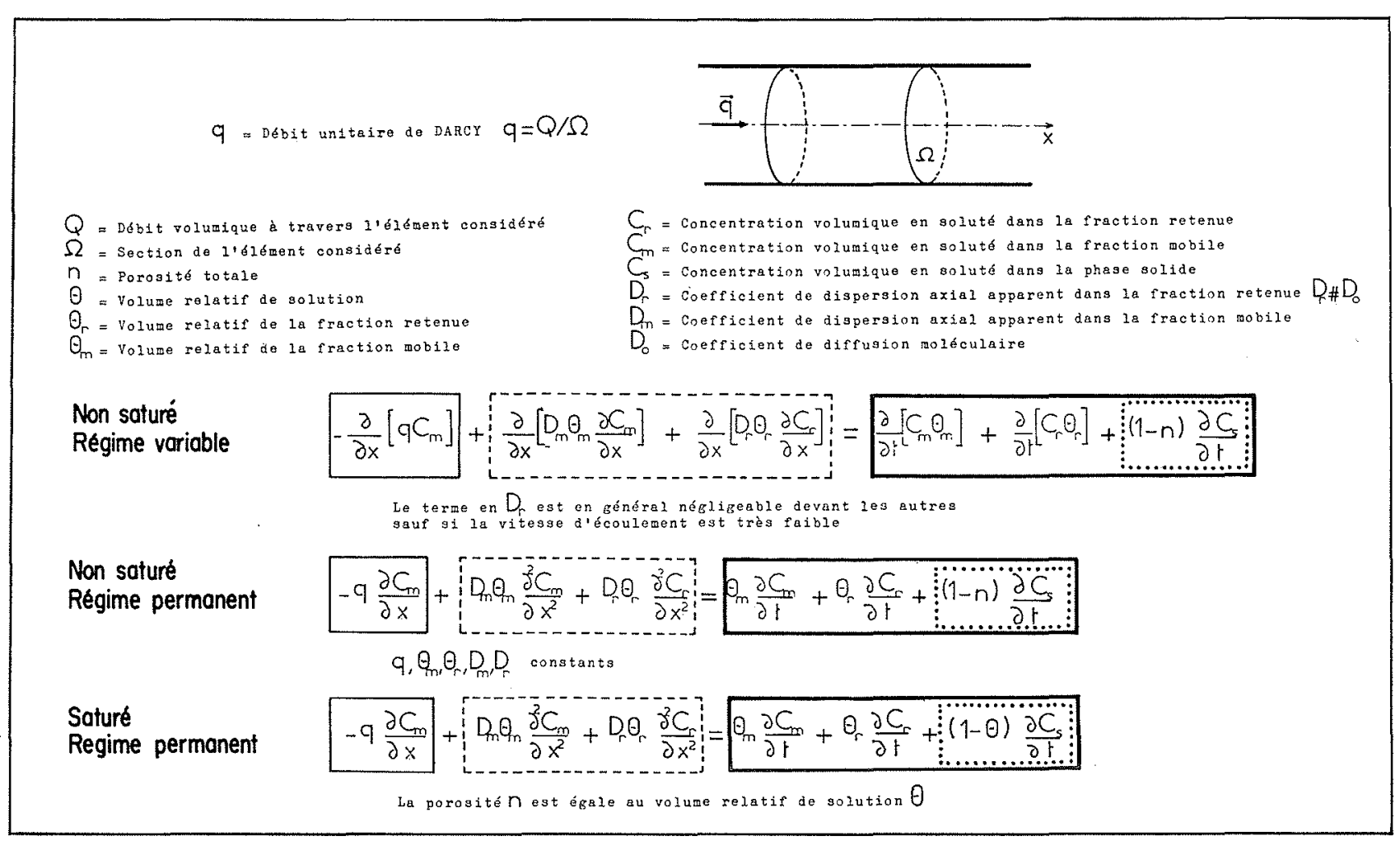


b) Hypothèse de la permanence du régime

En fait la plupart des modèles simples reposent sur l'hypothèse de la permanence du régime. Dans ce cas les conditions deviennent

$$
\begin{aligned}
& q=\text { constante } \\
& \theta_{m}=\text { constante } \\
& \theta_{r}=\text { constante } \\
& \theta=\text { constante } \\
& D_{m}=\text { constante }
\end{aligned}
$$

Nous obtenons alors l'expression suivante

$$
\begin{aligned}
&-q \frac{\partial C_{m}}{\partial x}+D_{m} \theta_{m} \frac{\partial^{2} C_{m}}{\partial x^{2}}+D_{r} \theta_{r} \frac{\partial^{2} C_{r}}{\partial x^{2}}= \\
& \theta_{m} \frac{\partial C_{m}}{\partial t}+\theta_{r} \frac{\partial C_{r}}{\partial t}+(1-n) \frac{\partial C_{s}}{\partial t}
\end{aligned}
$$

c) Expression analy tique globale en milieu saturé

En milieu saturé, la porosité $n$ est égale au volume relatif de solution $\theta$ ce qui revient à modifier uniquement le dernier terme de l'expression analytique.

\subsubsection{Considérations sur le terme dispersif}

a) Expression analytique du coefficient de dispersion en milieu poreux

La plupart des auteurs prennent en considération trois facteurs pour exprimer le coefficient de dispersion axiale dans un tube élémentaire représentatif du milieu poreux.

L'expression proposée par Aris (1959) [in Villermaux (1969] est représentée figure 14 .

Lorsque la vitesse intersticielle $u$ devient faible, cette expression se réduit évidemment à :

$$
D \simeq \frac{D_{o}}{T_{A}}
$$

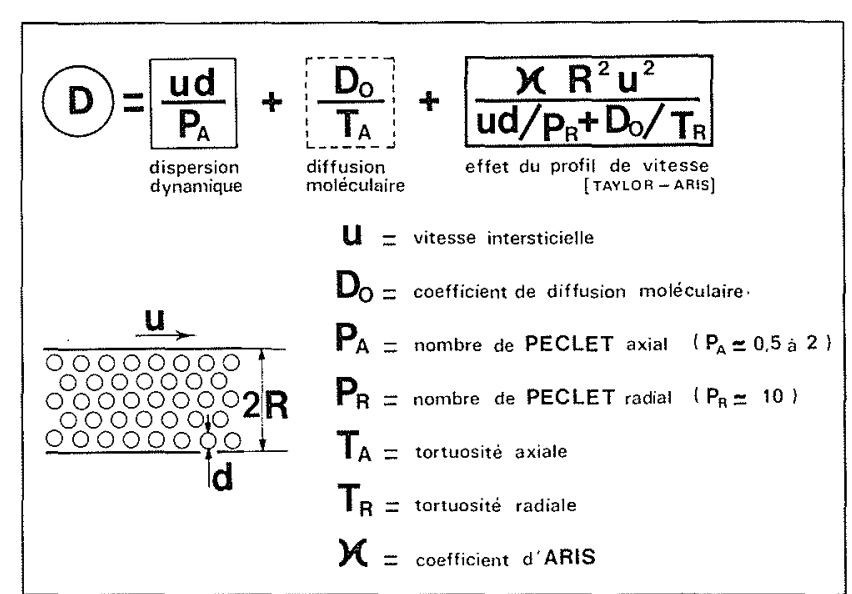

$14 /$ Coefficient de dispersion axiale en milieu poreux

Lorsque $u$ augmente, le terme prépondérant étant $u d / P_{A}$, la relation entre $D$ et $u$ est linéaire dans un domaine de vitesse extrêmement étendu [cf diagramme de Pfannkuch (1962) figure 9 ].

b) Différents cas de simplification du terme de dispersion dans l'expression analytique globale ( $c f$. tableau $n^{\circ} 2$ )

\section{b.a.) La vitesse intersticielle est élevée}

La dispersion du soluté dans la fraction mobile $\left(D_{m}\right)$ devient prépondérante et on peut négliger le terme en $D_{r}$ dans l'équation générale qui devient :

$$
\begin{aligned}
& -q \frac{\partial C_{m}}{\partial x}+D_{m} \theta_{m} \frac{\partial^{2} C_{m}}{\partial x^{2}}= \\
& \theta_{m} \frac{\partial C_{m}}{\partial t}+\theta_{r} \frac{\partial C_{r}}{\partial t}+(1-n) \frac{\partial C_{s}}{\partial t}
\end{aligned}
$$

Tableau 2

Expressions simplifiées de propagation en milieu poreux saturé

\section{EXPRESSION GENERALE}

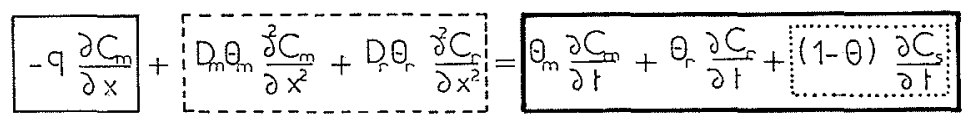

\section{SIMPLIFICATIONS}

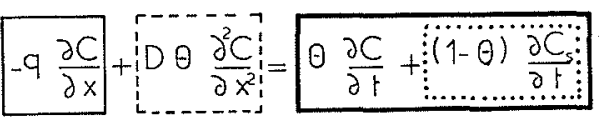

on ne distingue pas les 2 fractions du liquide

$$
-9 \frac{\partial C}{\partial x}+D \theta \frac{\partial^{2} C}{\partial x^{2}}: \theta \frac{\partial C}{\partial t}
$$

on néglige les processus de sorption

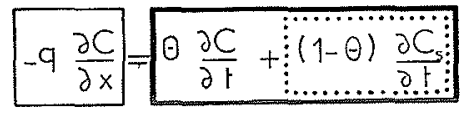

On néglige la dispersion 


\section{b.b.) la vitesse intersticielle est faible}

Comme nous venons de le voir en 4.2.6a) la dispersion axiale du soluté dans la fraction mobile devient du même ordre de grandeur que sa dispersion $D$, dans la fraction liée.

Il ne convient plus de distinguer les deux fractions du fluide, le transport du soluté étant le même dans ces deux fractions. Tout se passe comme si tout le soluté s'écoulait avec la même vitesse.

L'expression globale s'écrit alors

$$
-q \frac{\partial C}{\partial x}+D \theta \frac{\partial^{2} C}{\partial x^{2}}=\theta \frac{\partial C}{\partial t}+(1-n) \frac{\partial C_{s}}{\partial t}
$$

C'est l'expression la plus fréquemment adoptée par la plupart des auteurs et en fait, certains auteurs négligent même le terme de sorption et utilisent l'expression très simplifiée :

$$
-q \frac{\partial \mathcal{c}}{\partial x}+D \theta \frac{\partial^{2} C}{\partial x^{2}}=\theta \frac{\partial C}{\partial t}
$$

Citons l'expression proposée par Kyte (1973) qui néglige la dispersion :

$$
-q \frac{\partial C}{\partial x}=\theta \frac{\partial C}{\partial t}+(1-n) \frac{\partial C_{s}}{\partial t}
$$

\section{Explicitation du terme de rétention suivant la nature des processus physico-chimiques}

\subsection{TABLEAU SYNOPTIQUE DES DIFFERENTES AP- PROCHES DE L'INTERACTION}

Les processus physico-chimiques d'interaction se traduisent par des compétitions entre réactions de vitesses différentes conduisant à des états d'équilibre caractérisés par des isothermes.

La complexité de ces phénomènes a conduit les différents auteurs à formuler diverses hypothèses simplificatrices.

L'ensemble de ces tentatives est rassemblé dans le tableau synoptique suivant, figure 15 , qui distingue les processus selon la nature des cinétiques d'atteinte à l'équilibre et l'allure des isothermes.

\subsection{PROCESSUS REVERSIBLES}

Quelle que soit sa forme, l'isotherme d'équilibre, peut s'exprimer par une relation du type :

$$
C_{s(e q)}=f\left(C_{e q}\right)
$$

où $C_{e q}$ représente, selon le modèle hydrodynamique adopté, $C_{m(e q)}, C_{r(e q)}$ ou $C_{(m+r) e q}$

Le terme de sorption s'écrit alors selon la nature de la cinétique et la forme de l'isotherme retenues.

\subsubsection{Nature des cinétiques}

\section{a) Cinétique instantanée}

On suppose qu'à chaque instant l'équilibre est atteint et qu'en tout point :

$$
\frac{\partial C_{s}}{\partial t}=\frac{\partial}{\partial t}[f(\mathrm{C})]
$$

\section{b) Cinétique d'ordre 1}

La vitesse d'adsorption est proportionnelle à la concentration $C$ en phase liquide, et la vitesse de désorption à la concentration $C_{s}$ en phase solide; la vitesse globale s'écrit alors :

$$
\frac{\partial C_{s}}{\partial t}=k_{a} C-k_{d} C_{s}
$$

lorsque l'équilibre est atteint $\frac{\partial C_{s}}{\partial t}=0$, d'où $C_{s(e q)}=\frac{k_{q}}{k_{d}} C_{e q}$ équation de l'isotherme

\section{c) Cinétique d'ordre supérieur à l'unité}

$\mathrm{La}$ réaction d'interaction est :

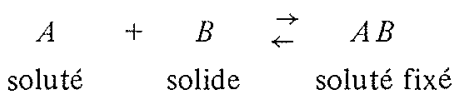

Gupta et Greenkorn (1973) retiennent une cinétique de réaction d'ordre 2 :

$$
\frac{\partial[A B]}{\partial t}=k_{a} \cdot[A] \cdot[B]-k_{d}[A B]
$$

[B] représente la quantité de solide encore accessible à la sorption.

Si $S$ est la capacité maximale

$$
[B]=S-C_{s}
$$

La cinétique s'écrit alors

$$
\frac{\partial C_{s}}{\partial t}=k_{a} \cdot C \cdot\left(S-C_{s}\right)-k_{d} C_{s}
$$

Lorsque l'équilibre est atteint $\frac{\partial C_{s}}{\partial t}=0$

$$
\frac{k_{a}}{k_{d}}=\frac{C_{s(e q)}}{C_{e q} \cdot\left(S-C_{s}\right)}
$$

En supposant que l'isotherme de sorption a la forme de Langmuir

$$
C_{s(e q)}=\frac{K \cdot S \cdot C_{e q}}{1+K C_{e q}}
$$

qui s'écrit encore

$$
K=\frac{C_{s(e q)}}{C_{e q} \cdot\left(S-C_{s}\right)_{e q}}
$$

qui n'est autre que la loi d'action de masse, on a

$$
k_{d}=\frac{k_{a}}{K}
$$

La cinétique s'écrit donc :

$$
\frac{\partial C_{s}}{\partial t}=k_{a}\left[C .\left(S-C_{s}\right)-\frac{1}{K} C_{s}\right]
$$

\subsubsection{Forme des isothermes}

a) Isothermes linéaires

Dans ce cas $C_{s(e q)}=m C_{e q}$, $m$ étant la pente de l'isotherme. 


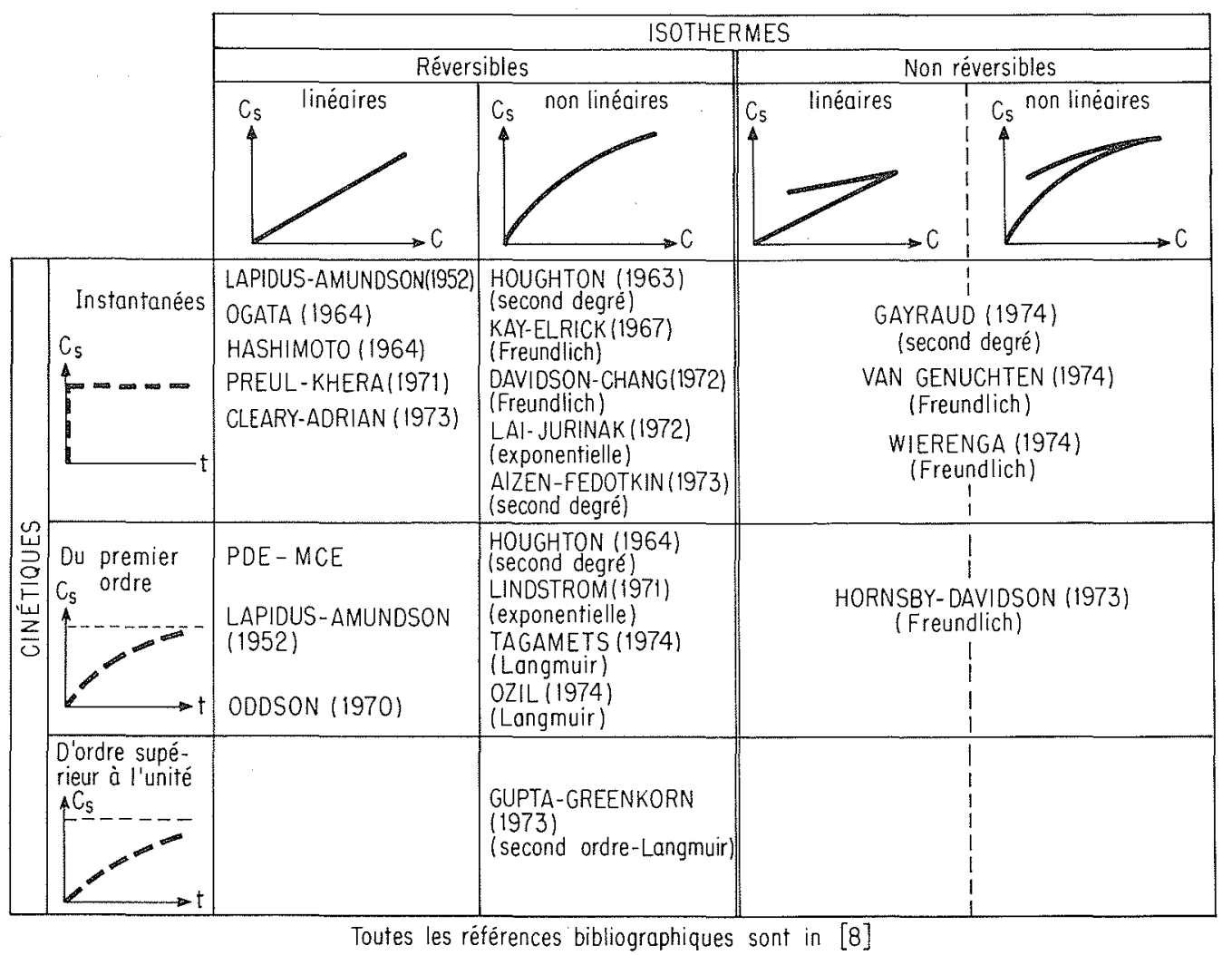

15/ Tableau synoptique des différentes approches de l'interaction

Si la cinétique est instantanée

$$
\frac{\partial C_{s}}{\partial t}=m \frac{\partial C}{\partial t}
$$

Si la cinétique est d'ordre 1

$$
\frac{\partial C_{s}}{\partial t}=k_{a}\left(C-\frac{C_{s}}{m}\right)
$$

b) Isothermes du second degré

Cette représentation de l'isotherme, proche de la linéarité, est retenue par différents auteurs [Houghton (1963) (1964) Aizen-Fedotkin-Marutovskii (1973)].

Le développement adopté est le suivant :

$$
C_{s(e q)}=k_{0}+k_{1} C_{e q}+k_{2} C_{e q}^{2}
$$

c) Autres représentations

D'autres représentations peuvent être proposées :

c.a) Isothermes de Langmuir [Tagamets (1974), Ozil (1974), Gupta-Greenkorn (1973)]

$$
C_{s(e q)}=\frac{K C_{e q}}{1+K C_{e q}}
$$

où $K$ est la constante d'équilibre (loi d'action de masse)

c.b) Isothermes de Freundlich [Kay-Elrick (1967), DavidsonChang (1972)].

$$
C_{s(e q)}=K C_{e q}^{\frac{1}{N}} \quad N \text { constante }>1
$$

c.c) Isothermes exponentielles [Lai-Jurinak (1972), Lindstrom (1971)] .

On trouvera le détail de ces expressions dans Rochon-Molinari (1974).

\subsection{PROCESSUS NON REVERSIBLES}

Les recherches de Warncke et Barber (1973) introduisent parallèlement à la notion d'isotherme d'adsorption, celle d'isotherme de désorption ce qui permet de mettre en évidence les fractions réversibles et irréversibles de la sorption.

A l'exception de Hornsby et de Davidson (1973) qui considèrent une cinétique du premier ordre autour de l'équilibre. représenté par un isotherme de Freundlich, avec:

$$
\frac{\partial C_{s}}{\partial t}=k_{d}\left[K C^{\frac{1}{N}}-C_{s}\right]
$$

cette approche est généralement traitée en supposant la cinétique instantanée comme le font : Van Genuchten, Davidson et Wierenga (1974) ainsi que Gayraud (1974).

Dans tous les cas, on considere que la fonction d'entrée dans le système se caractérise par un seul extrémum (fonctions d'entrée de type impulsionnelles) et que chaque tranche élémentaire est soumise successivement à un front de montée et à une phase de désorption.

On se trouve en présence de plusieurs relations

- une relation d'adsorption unique obéissant à la loi d'isotherme de sorption choisie

- une infinité de relations de désorption (à une date déterminée). A chaque section de la colonne de milieu poreux, correspond une relation de désorption particulière. Gayraud lui attribue, comme pour la sorption, l'équation dérivée de l'isotherme de désorption du second degré. Van Genuchten la caractérise par une équation de même type, mais avec une représentation de Freundlich pour l'isotherme de désorption.

Au cours de l'intégration, pour $t$ constant, on introduit alors dans l'équation du bilan, un terme de sorption dans les tranches ělémentaires affectées par le front de montée, et un terme 
de désorption dans les tranches élémentaires affectées par le front de descente. Les coefficients des termes de désorption dépendant évidemment des concentrations maximales ayant affecté les dites tranches.

\section{Choix d'un modèle pratique pour l'exploitation rapide d'essais exploratoires de laboratoire et de terrain}

\subsection{CRITERES DE CHOIX}

Avant toute considération, il ne faut pas oublier que le degré de raffinement d'un modèle mathématique doit être adapté à l'usage que l'on en attend.

Il doit être en particulier compatible avec la précision des clonnées expérimentales dont on dispose et avec celle des paramètres que l'on cherche à déterminer.

Le seul modèle capable de tenir compte à la fois des processus de sorption et de désorption ainsi que de la cinétique est le modèle de Hornsby-Davidson (1973)

La mise en oeuvre d'un tel modèle comme de celle des modèles simplifiés de Gayraud 1973), Van Genuchten (1974) et Wierenga (1975) implique un traitement numérique itératif d'un coût rhédibitoire.

En outre, notre préoccupation première consistant à appréhender les processus de propagation de l'eau au moyen de traceurs de la phase liquide peu affectés par les processus de sorption, il paraissait justifié de renoncer, en première approche, à ces modèles sophistiqués pour faire appel à des modèles plus simples faisant intervenir un nombre de paramètres moins élevé.

Parmi ces modèles simples, il nous a paru judicieux de puiser dans l'arsenal des modèles utilisés en Génie Chimique, modèles qui ont été mis à l'épreuve d'exploitation systématique. Notre choix s'est porté sur le modèle très connu des Mélangeurs en Cascade avec Echange (M.C.E.) qui présente l'avantage de se prêter à des ajustements très aisés.

\subsection{EQUIVALENCE DES MODELES MCE ET PDE}

Pour représenter la dispersion axiale avec échange de matière d'un écoulement, le génie chimique propose deux représentations simples et commodes : les modèles PDE et MCE.

\subsubsection{Modèle P.D.E.}

Ce modèle reprend les hypothèses hydrodynamiques du modèle simplifié donné en 4.2.6.b.b. (assimilation de la fraction retenue de la phase liquide avec la phase solide) et suppose que le transfert de masse est gouverné par une cinétique de transfert du premier ordre et une isotherme linéaire.

La solution analytique de ce système est donnée en fonction de 3 paramètres :

$$
\begin{array}{ll}
P=\frac{q L}{D \theta} & \begin{array}{l}
\text { critère de Peclet axial (paramètre de } \\
\text { dispersion) }
\end{array} \\
N=\frac{k L}{u} \frac{1-\theta}{\theta} & \begin{array}{l}
\text { nombre d'unités de transfert (para- } \\
\text { mètre cinétique) }
\end{array} \\
R=\frac{\theta}{\theta+m(1-\theta)} & \begin{array}{l}
\text { critère de rétention (paramètre } \\
\text { d'équilibre) }
\end{array}
\end{array}
$$

\subsubsection{Modèle M.C.E.}

Ce modèle repose sur les mêmes hypothèses d'interaction mais l'écoulement axial est, dans ce cas, représenté par une cascade de $J$ cellules identiques en série contenant chacune les phases mobile et stationnaire supposées de composition uniforme à chaque instant (figure 16).

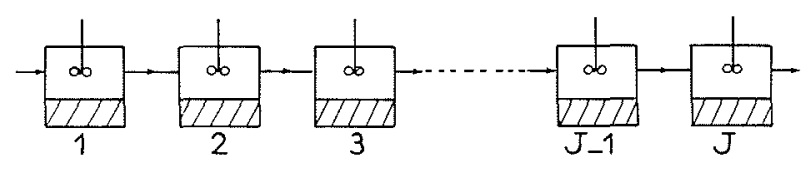

$16 /$

Ecrivons le bilan de matière dans la cellule $i$

$$
\begin{aligned}
& q \theta C_{i-1}=q \theta C_{i}+\frac{V}{J} \theta \frac{d C_{i}}{d t}+\frac{V}{J} \theta \frac{d C_{s i}}{d t} \\
& \frac{d C_{s i}}{d t}=k\left(C_{i}-\frac{C_{s i}}{m}\right)
\end{aligned}
$$

$\frac{V}{J}=$ volume d'une cellule $V=$ volume de la colonne équivalente

La solution analytique de ce système est donnée en fonction des 3 paramètres :

$$
\begin{array}{ll}
J & \begin{array}{l}
\text { nombre de cellules (paramètre de } \\
\text { dispersion) }
\end{array} \\
R=k \frac{V}{q} \frac{(1-\theta)}{\theta} & \begin{array}{l}
\text { nombre d'unités de transfert (para- } \\
\text { mètre cinétique) }
\end{array} \\
R=\frac{\theta}{\theta+m(1-\theta)} & \begin{array}{l}
\text { critère de rétention (paramètre } \\
\text { d'équilibre). }
\end{array}
\end{array}
$$

\subsubsection{Condition d'équivalence}

Lorsque le nombre de cellules $(J)$ est grand, il est facile de montrer que ces modèles (P.D.E. et M.C.E.) sont équivalents pour :

$$
P=2 J
$$

Le choix préférentiel du modèle M.C.E., discontinu, n'est dicté que par la commodité de traitement mathématique, les différences étant, dans les conditions habituellement rencontrées, insignifiantes.

\subsection{EQUIVALENCE DU P.D.E. ET DU MODELE PROPOSE PAR GAUDET}

\subsubsection{Hypothèses communes aux deux modèles}

- La phase liquide est incompressible et continue

- L'écoulement est unidirectionnel et isotherme

- La matrice solide est indéformable

- Le régime est permanent.
6.3.2. Hypothèses complémentaires du modèle proposé par Gaudet
- Si le milieu n'est pas saturé, la phase gazeuse est à pression constante
- Il n'y a pas d'interaction avec le solide
- Le transfert de matière entre les fractions mobile et retenue 
de la solution est régi par l'équation d'échange proposée par Deans (1963) et Coats (1964).

Le système d'équations à résoudre est donc :

$$
\left\{\begin{array}{l}
-q \frac{\partial C_{m}}{\partial x}+D_{m} \theta_{m} \frac{\partial^{2} C_{m}}{\partial x^{2}}=\theta_{m} \frac{\partial C_{m}}{\partial t}+\theta_{r} \frac{\partial C_{r}}{\partial t} \\
\frac{\partial C_{r}}{\partial t}=\alpha \cdot\left(C_{m}-C_{r}\right)
\end{array}\right.
$$

\subsubsection{Hypothèses complémentaires du modèle P.D.E.}

- le milieu est saturé

- la phase liquide est composée d'une seule fraction réagissant avec le solide. Le transfert de matière est gouverné par une cinétique du premier ordre et une isotherme de partage linéaire.

Le système d'équations à résoudre est donc :

$$
\left\{\begin{array}{c}
-q \frac{\partial C}{\partial x}+D \theta \frac{\partial^{2} C}{\partial x^{2}}=\theta \frac{\partial C}{\partial t}+(1-\theta) \frac{\partial C_{s}}{\partial t} \\
\frac{\partial C_{s}}{\partial t}=k\left(C-\frac{C_{s}}{m}\right)
\end{array}\right.
$$

\subsubsection{Equivalence formelle [Villermaux (1975)]}

Nous pouvons constater que ces modèles, ne reposant pas sur les mêmes hypothèses et ne tenant donc pas compte des mêmes phénomènes, sont formellement identiques. En identifiant les deux systèmes nous trouvons:

\begin{tabular}{|c|c|}
\hline Modèle Gaudet & Modèle P.D.E. \\
\hline$C_{m}$ & $\longrightarrow$ \\
$C_{r} \longrightarrow \frac{C}{m}$ \\
$\theta_{m} \longrightarrow \theta$ \\
$\alpha \theta_{r} \longrightarrow k(1-\theta)$ \\
$D_{m} \longrightarrow D$ \\
\hline
\end{tabular}

\subsection{EXPLOITATION DU MODELE M.C.E.}

Comme la plupart des modèles, le M.C.E. peut être traité principalement de 4 façons différentes.

\subsubsection{Utilisation de la solution analytique}

Connaissant la solution analy tique (Villermaux 1972), on peut déterminer les valeurs optimales des 3 paramètres $N, J, R$ par une méthode de recherche directe de régression non linéaire à partir de la courbe expérimentale.

\subsubsection{Utilisation de la méthode des moments}

Rappelons qu'il s'agit de calculer les paramètres du modèle en confrontant les valeurs expérimentales des moments aux expressions théoriques qu'on obtient facilement à partir de la seule connaissance de la transformée de Laplace de la distribution [Villermaux (1969), Lees (1969)].

Cette méthode s'avère lourde et imprécise en raison notamment du poids considérable attribué dans les calculs aux mesures expérimentales affectées de la plus grande incertitude (quelues de courbes). Ceci exclut en pratique le recours à des moments d'ordre supérieur à 2 .

\subsubsection{Utilisation de la fonction de transfert}

La fonction de transfert d'un système est la transformée de Laplace de la réponse impulsionnelle $E(t)$ à une impulsion de Dirac.

$$
G(s)=\mathscr{e}[E(t)]=\int_{0}^{\infty} e^{-s t} \cdot E(t) \cdot d t
$$

$G(s)$ est calculable expérimentalement à partir d'un couple quelconque de signaux entrée-sortie, soient $C_{1}(t)$ et $C_{2}(t)$

$$
G(s)=\frac{\mathfrak{E}\left[C_{2}\right]}{\mathfrak{f}\left[C_{1}\right]}=\frac{\bar{C}_{2}}{\bar{C}_{1}}
$$

Connaissant $G$ nous avons

$$
F=\frac{J}{s}\left[\left(\frac{1}{G}\right)^{\frac{1}{T}}-1\right]
$$

Posons $Y=(1-F)^{-1} \quad$ et $X=\frac{1}{s}$

Le système (1) devient

$$
Y=(1-R)^{-1}+N X(1-R)^{-2}
$$

En traçant cette droite nous obtenons immédiatement $N$ et $R$ par la pente et l'ordonnée à l'origine [Villermaux (1962)].

\subsubsection{Utilisation de critères de forme}

Les méthodes précédentes de traitement sont bien connues mais souvent inapplicables par le praticien ne disposant pas d'un ordinateur et voulant exploiter l'information contenue dans une courbe expérimentale.

Villermaux (1972) a sélectionné des critères graphiques sensibles aux variations des 3 paramètres et a établi des corrélations les estimant. Les abaques qu'il fournit permettent d'évaluer l'ordre de grandeur de ces paramètres et il ne convient pas d'y rechercher une grande précision.

\section{Etude expérimentale}

\subsection{CONDUITE DES INVESTIGATIONS}

\subsubsection{Nature des essais}

La démarche adoptée implique trois niveaux d'investigations : a) 1 er niveau : étude statique en laboratoire, de la nature des mécanismes de rétention des substances sur des substrats, par obtention des cinétiques et des isothermes de sorption et de désorption.

b) 2ème niveau : étude dynamique, en laboratoire (par percolation sur colonnes), pour établir les équations de bilan et faire choix des paramètres caractérisant les processus hydrodynamiques de transfert et physico-chimiques de sorption des mêmes systèmes biphasiques.

c) Bème niveau : étude en vraie grandeur sur site expérimental, afin d'apprécier l'importance et la nature des correctifs susceptibles d'intervenir dans une transposition "laboratoire-terrain".

\subsubsection{Organisation et déroulement des essais}

L'organisation et le déroulement des essais ont été articulés sur la possibilité de disposer d'une parcelle expérimentale (parcelle 
de Bonnaud implantée dans une plaine alluviale du Jura) aménagée conjointement par le Bureau de Recherches Géologiques et Minières (BRGM) et le C.E.A. dans le cadre d'une Action Thematique Programmée (A.T.P.) du C.N.R.S.

Les essais de terrain relatifs à la présente étude consistaient en l'obtention de réponses impulsionnelles de diverses substances entre un dipôle implanté sur une ligne d'écoulement d'une nappe captive en circulation forcée assurée grâce à un dispositif déjà décrit (Molinari-Peaudecerf 1975).

Les essais de laboratoire ont porté sur :

- un échantillon moyen représentatif de ce même terrain de Bonnaud

- un matériau alluvionnaire faisant par ailleurs l'objet d'investigations approfondies à l'E.N.S. d'Hydraulique de Grenoble et obligeamment fourni par MM. Gaudet et Vachaud

- des matériaux modèles pouvant être considérés comme éléments constitutifs des terrains alluvionnaires étudiés, à savoir :

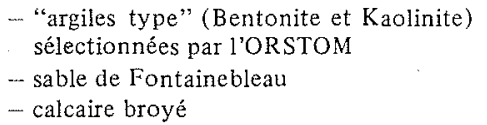

La ventilation de l'ensemble des essais, qui à leur achèvement doivent convrir la gamme des traceurs usuels de l'eau et une série de substances polluantes représentatives, peut être condensée dans le schéma suivant :

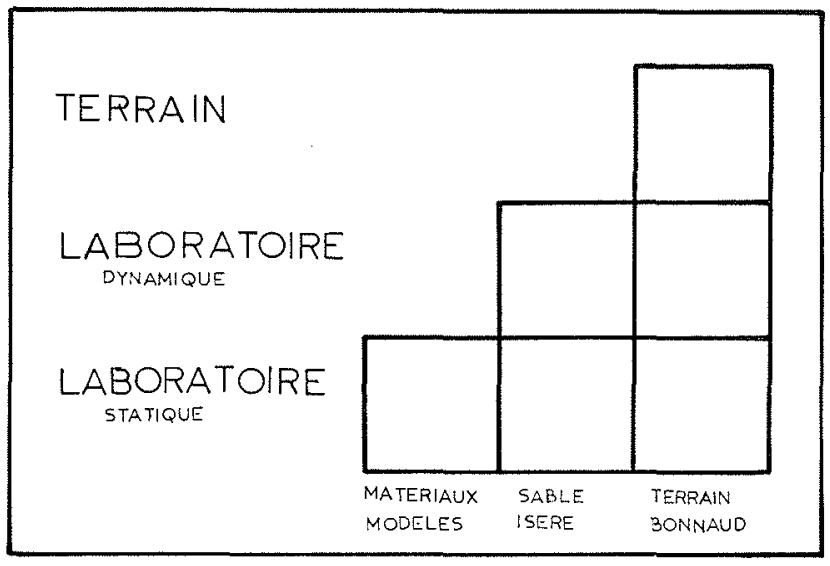

$17 /$

Pour des raisons techniques inhérentes aux contraintes d'expérimentation sur le terrain, les essais de terrain ont anticipé sur les mesures de laboratoire correspondantes et les résultats dont nous disposons restent encore très fragmentaires.

\subsection{RESULTATS EXPERIMENTAUX}

\subsubsection{Isothermes}

Ces isothermes ont été obtenues pour des gammes de concentration couramment rencontrées en hydrologie.

Les difficultés d'obtention des isothermes nous ont conduits à débuter par les substances dont on maitrise le mieux la mesure, c'est-à-dire les substances fluorescentes. Leur gamme extrêmement étendue comprend des bons traceurs de l'eau (Uranine) et des substances fortement sorbées (telle la Rhodamine B).

La figure 18 met en évidence la grande différence de comportement de ces deux traceurs sur le sable alluvial de l'Isère. L'isotherme de l'Uranine a une forme de Langmuir très mar-

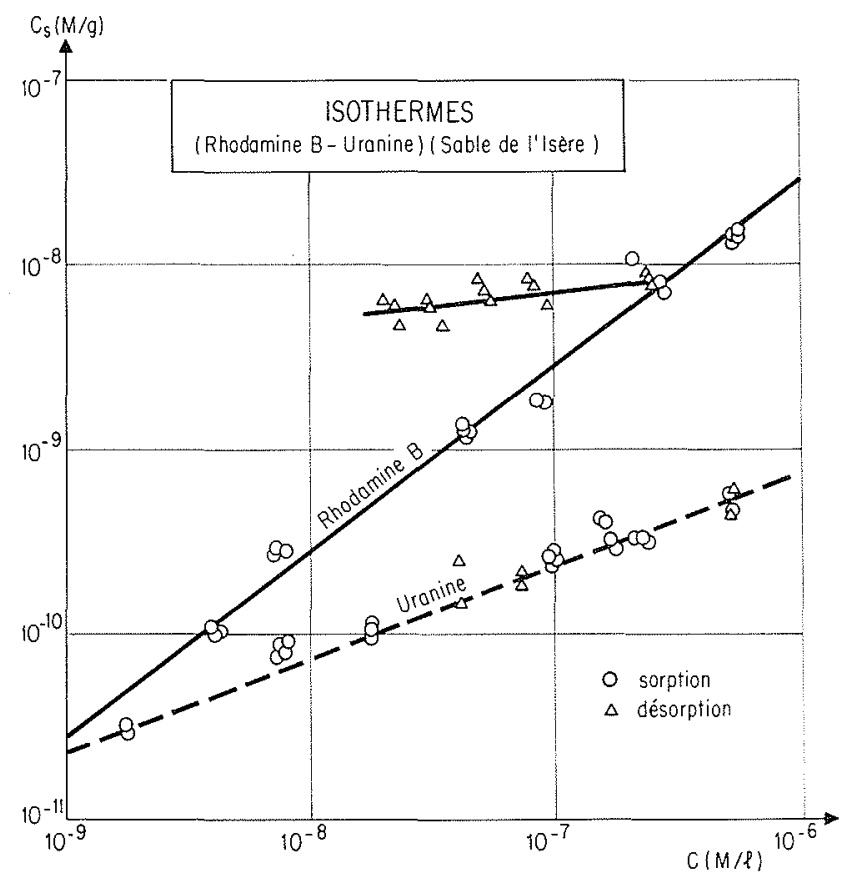

$18 /$ Isothermes de sorption et de désorption (Rhodamine B - Uranine) (Sable de I'Isere)

quée et présente une excellente réversibilité, tandis que l'isotherme de la Rhodamine $\mathrm{B}$ dans le domaine étudié révèle une sorption considérable et, de surcroit, en grande partie irréversible.

La figure 19 permet de comparer les comportements sur le même matériau de la famille des quatres colorants xanthéniques.

D'autres essais ont montré que les isothermes de ces mêmes substances se révèlent très différentes d'un matériau à l'autre.

\subsubsection{Distribution des temps de séjour sur colonnes}

Toutes ces distributions de temps de séjour ont été obtenues directement par injections instantanées sur un dispositif expérimental obligeamment mis à notre disposition par l'ENSH de Grenoble et décrit par Gaudet (1974).

Avant chaque essai, la colonne était préalablement saturée. Pour certaines substances, on a pris soin de renouveler ces essais dans différentes conditions hydrodynamiques.

La figure 20 met en évidence les grandes différences de comportement de substances utilisées pour le traçage en hydrologie (on notera que l'un des traceurs, la Rhodamine B, n'est pas restitué).

\subsubsection{Distribution des temps de séjour sur le terrain}

Les premiers résultats de ces essais actuellement en cours sont représentés figure 21 . On constate ici encore des différences de comportement extrêmement significatives.

\subsection{INTERPRETATION DES PREMIERS RESULTATS}

\subsubsection{Composition de l'isotherme d'un matériau à partir des} isothermes de ses principaux constituants

Cette reconstitution a été effectuée avec succès pour la plupart des colorants xanthéniques dans le cas du sable de l'Isère. 


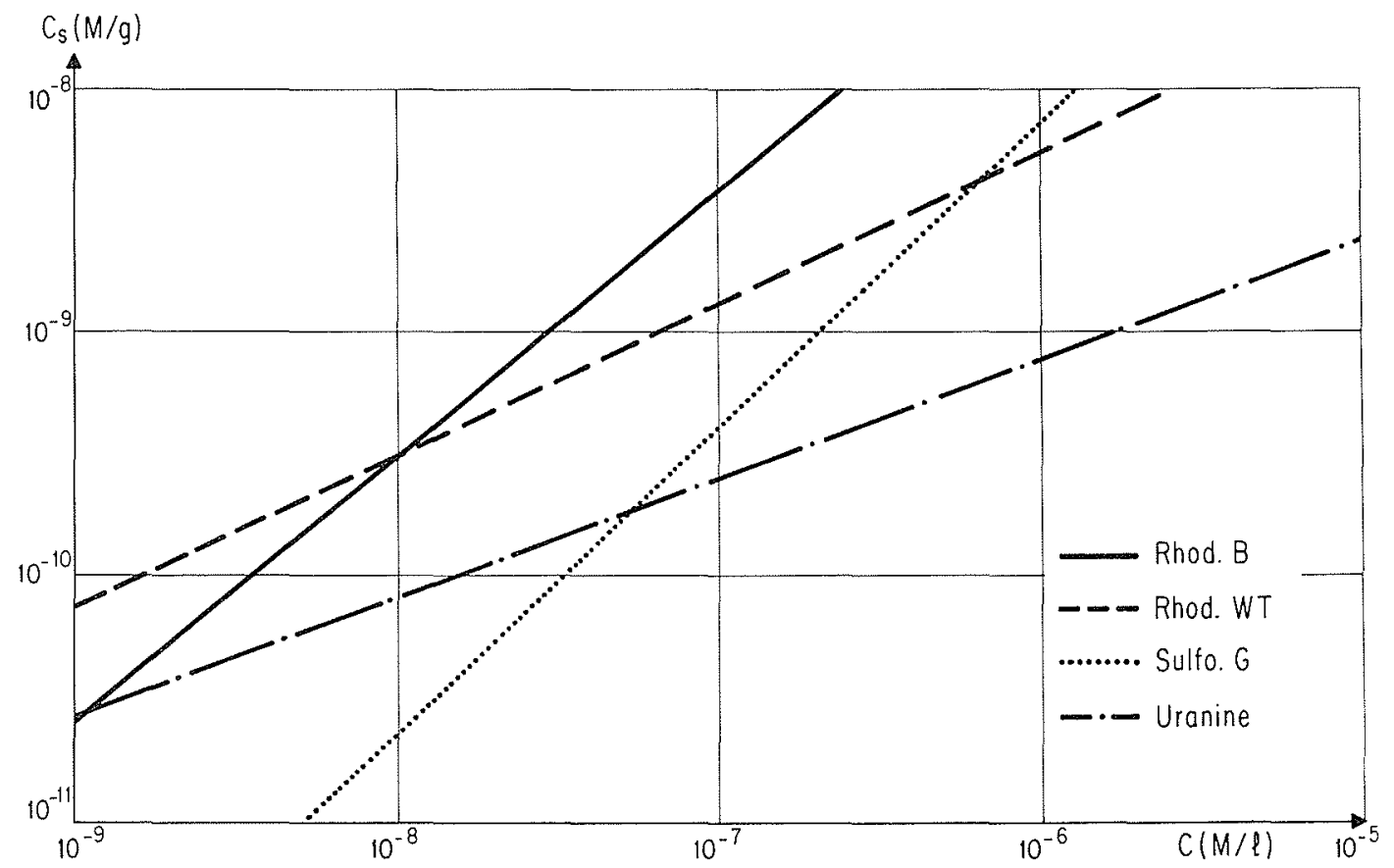

$19 /$ Isothermes de sorption. Sable de I'Isère

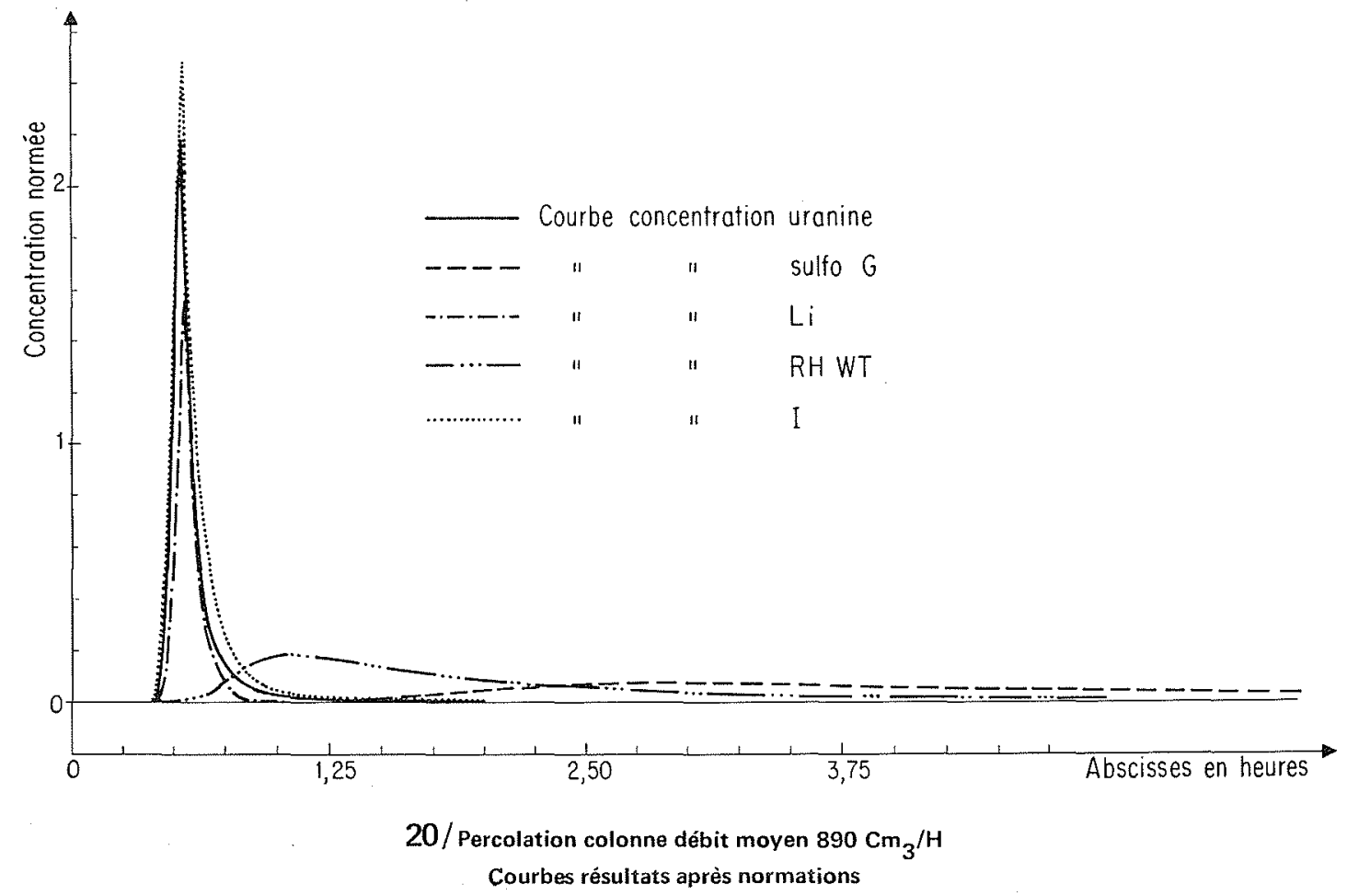




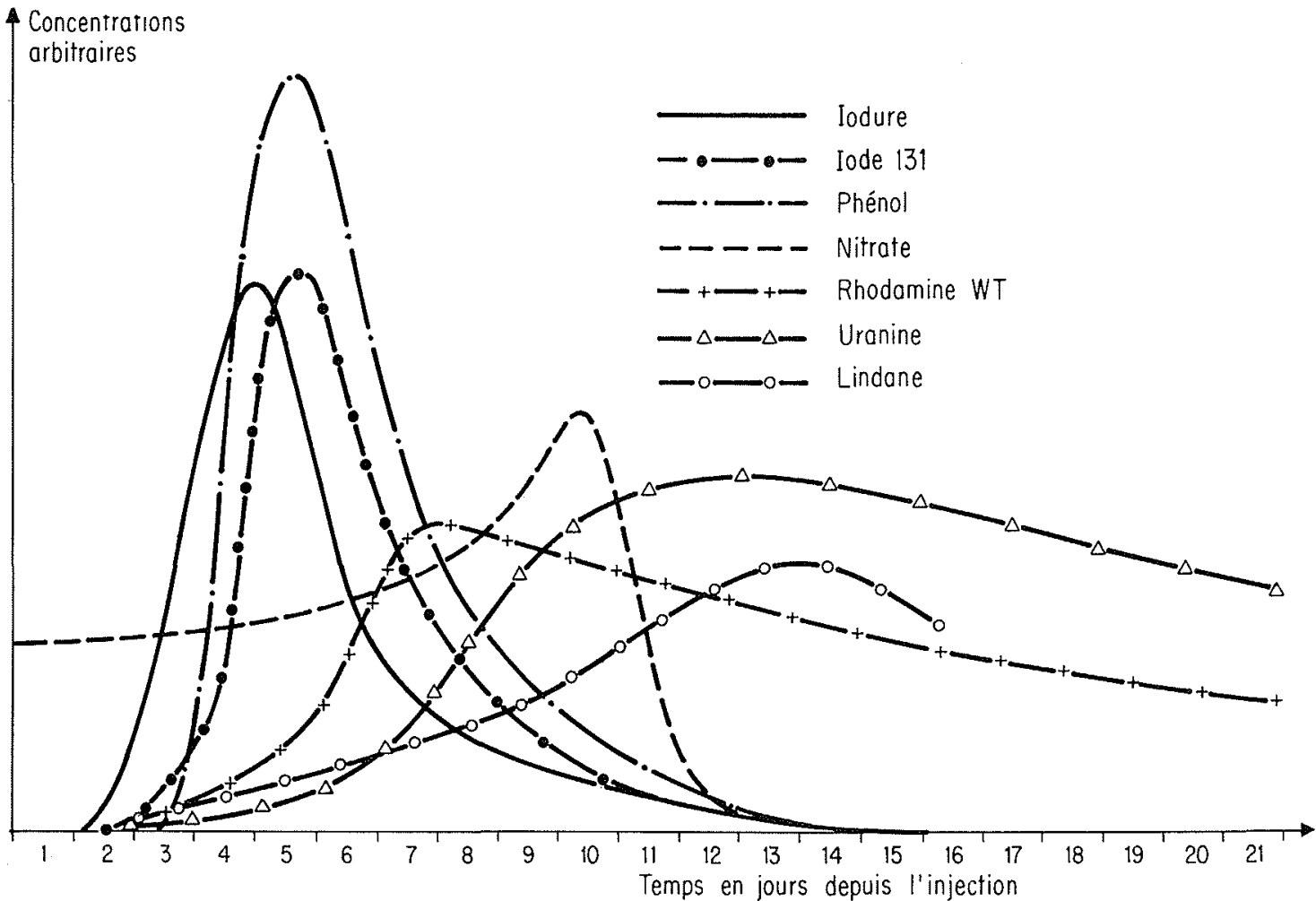

$21 /$ Réponses impulsionnelles sur parcelle expérimentale

Dans ces évaluations, on a tenu compte de la forme exponentielle des isothermes (assimilées à des isothermes de Freundlich), de la composition minéralogique du matériau, des surfaces spécifiques des constituants-type et du matériau, comme le schématise la figure 22 .

Ces premiers résultats confirment l'additivité des effets de chacun des constituants considéré isolément.
7.3.2. Ajustement des réponses impulsionnelles obtenues par des essais dynamiques sur colonne au modèle M.C.E.

La figure 23 précise les relations entre les paramètres du modèle et les données expérimentales. Les ajustements ont été effectués par la méthode des critères de forme, décrite en 6.4 .4

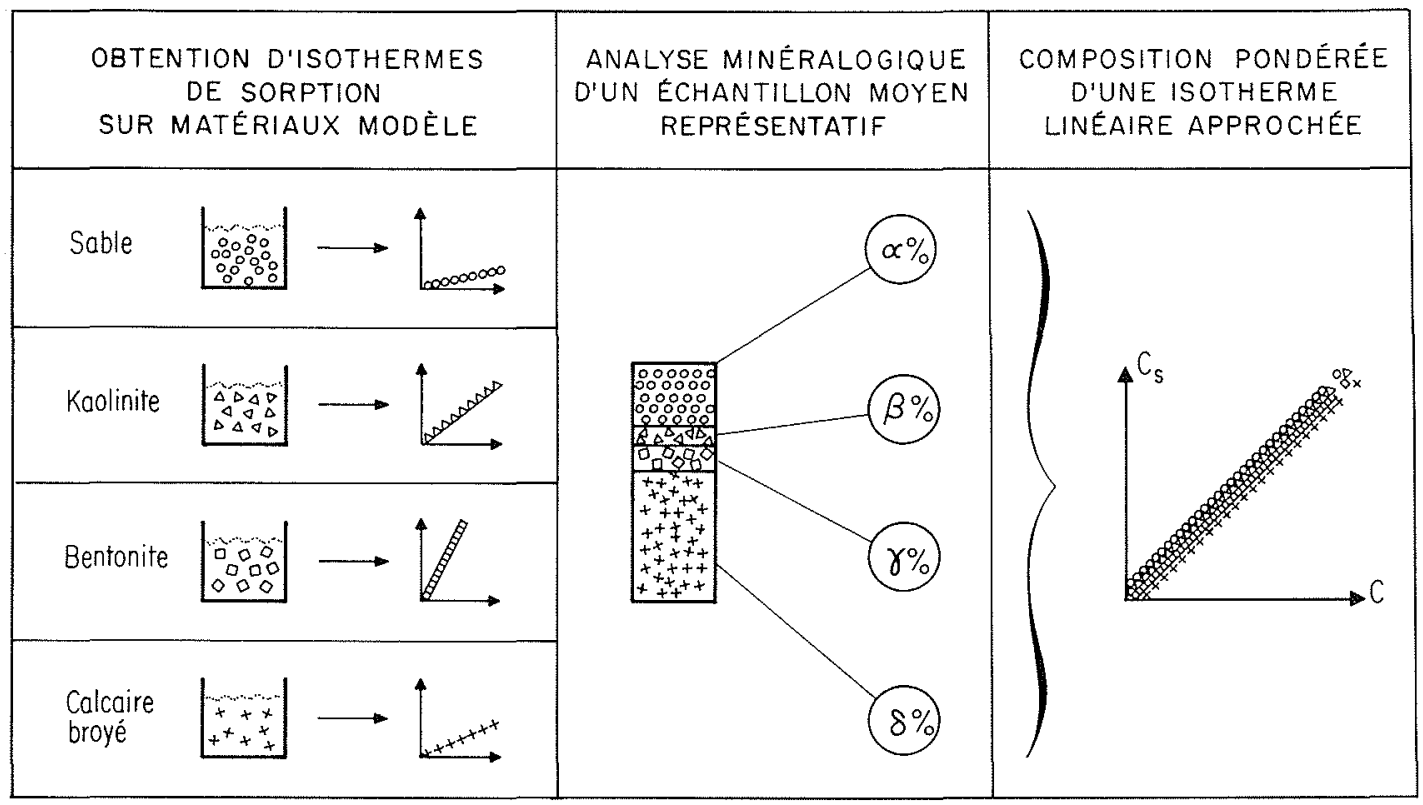

22/ Obtention des caractéristiques de sorption (isothermes) d'un terrain à partir de l'analyse de ses constituants majeurs 


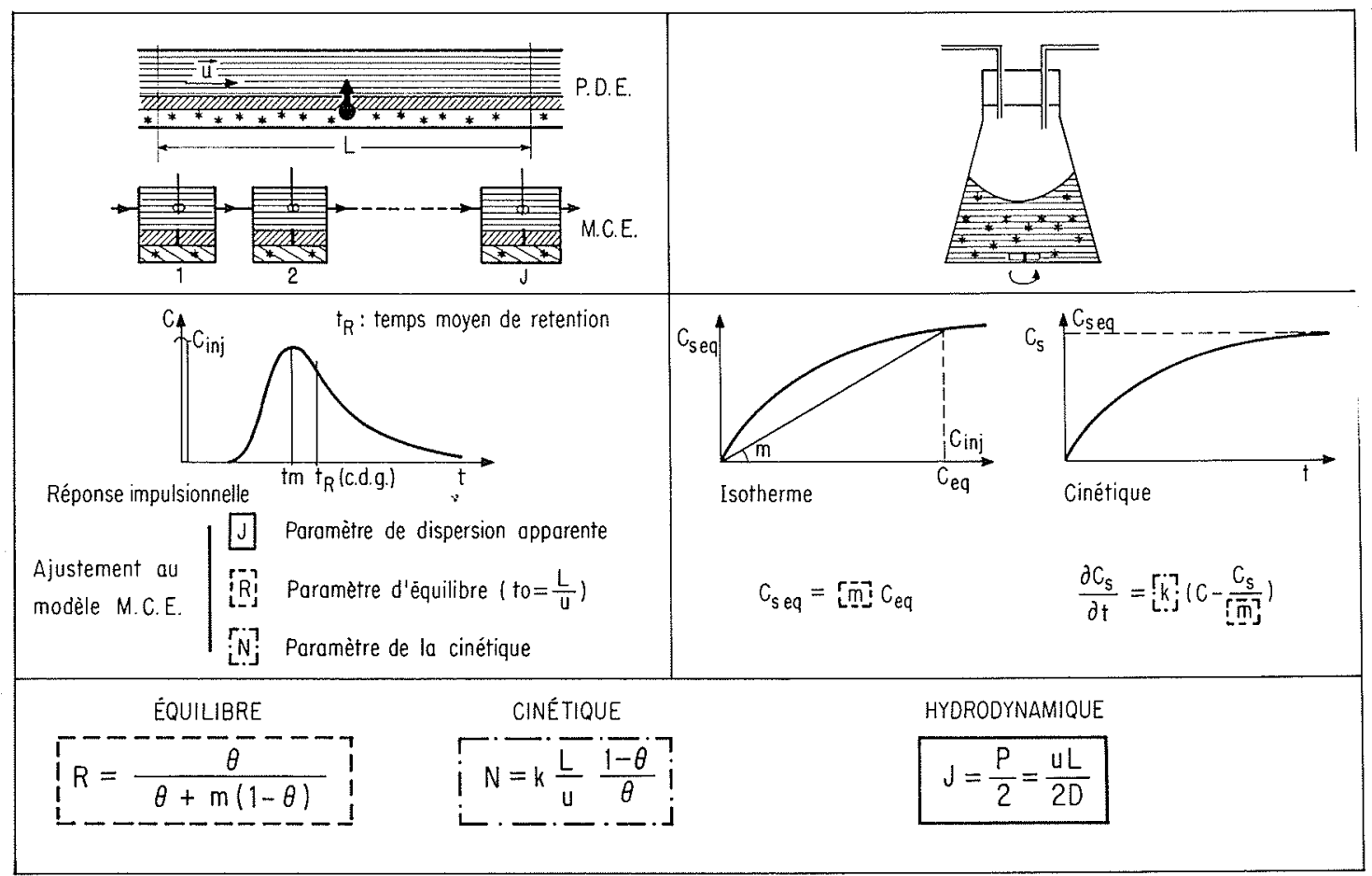

23/ Exploitation du modèle M.C.E.

Le tableau suivant (figure 24) donne les premiers résultats de ces ajustements qui confirment la valeur élevée du paramètre $(J)$ pour tous les essais.

\begin{tabular}{|c|c|c|c|c|c|c|c|}
\hline & $\mathrm{cm} \mathrm{h}^{u}$ & $\theta$ & $\begin{array}{l}t_{0} \\
\min \end{array}$ & $\begin{array}{c}t_{R} \\
\text { min }\end{array}$ & $R$ & $J$ & $N$ \\
\hline \multirow{3}{*}{ Uranine } & 60,6 & 0,38 & 39,9 & 46,8 & 0,85 & 42 & 0,6 \\
\hline & 19,4 & 0,36 & 124,7 & 149,4 & 0,83 & 51 & 1,6 \\
\hline & 86,3 & 0,35 & 28 & 30,5 & 0,92 & 40 & 0 \\
\hline \multirow{3}{*}{$\begin{array}{c}\text { Rhodamine } \\
\text { Wt }\end{array}$} & 60,6 & 0,38 & 39,9 & 130,8 & 0,3 & 40 & 1 \\
\hline & 21,4 & 0,36 & 112,8 & 581 & 0,2 & 47 & 1,4 \\
\hline & 82,7 & 0,36 & 29,3 & 108.4 & 0,27 & 37 & 0,6 \\
\hline \multirow{2}{*}{$\begin{array}{c}\text { Sulforhodamine } \\
G\end{array}$} & 18,9 & 0,36 & 127,8 & 398,6 & 0,03 & 47 & 2,2 \\
\hline & 82,8 & 0,36 & 29,2 & 242,4 & 0,12 & 37 & \\
\hline Rhodamine $\mathrm{B}$ & 83 & 0,36 & 29,2 & \multicolumn{4}{|c|}{ pas de restitution } \\
\hline Iodure & 82,6 & 0,36 & 29,3 & 30,8 & 0,95 & 42 & 0,3 \\
\hline Lithium & 82 & 0,36 & 29,5 & 37,5 & 0,79 & 41 & 1,7 \\
\hline Nitrate & 85,7 & 0,35 & 28,2 & 30 & 0,94 & 45 & 0 \\
\hline Phénol & 85,8 & 0,35 & 28,2 & 31,4 & 0,9 & 39 & $\infty$ \\
\hline Cuivre & 87,1 & 0,35 & 27.8 & \multicolumn{4}{|c|}{ pas de restitution } \\
\hline
\end{tabular}

$t_{0}=$ temps de rétention moyen d'un soluté non retenu

$t_{R}=$ temps de rétention moyen de la substance considérée

\section{4/ Ajustement au modèle MCE des essais sur colonne de sable de I'lsère.}

On remarque que la valeur de $(R)$, indépendante de la vitesse, est d'autant plus élevée que la restitution de la substance est rapide (pour un soluté non retenu, on a $R=1$ ).

La valeur du paramètre $(N)$, caractéristique de la cinétique d'échange, est extrêmement faible pour l'iodure dont les taux et vitesse de restitution sont les plus élevés.

\subsubsection{Comparaison des essais statiques et dynamiques}

Cette comparaison peut être effectuée directement en confrontant les valeurs respectives de $(R)$ obtenues par ajustement du modèle et de $(m)$ pente de l'isotherme (cf tableau figure 25 ).

\begin{tabular}{|l|c|c|c|}
\cline { 2 - 4 } \multicolumn{1}{c|}{} & \multicolumn{2}{|c|}{ STATIQUE } & DYNAMIQUE \\
\cline { 2 - 4 } & $\begin{array}{c}\text { Isotherme } \\
\text { expérimentale }\end{array}$ & $\begin{array}{c}\text { Isotherme } \\
\text { obtenue par composition }\end{array}$ & $\begin{array}{c}\text { M.C.E. } \\
\text { sur colonne }\end{array}$ \\
\hline & $m=\frac{C_{s}}{C_{\text {inj }}}(\mathrm{M} / 1$ de terrain) \\
\hline Uranine & $m=\frac{1-R}{R} \frac{0}{1 \cdots \theta}$ \\
\hline Rhodamine $W T$ & 1,6 & 0,23 & 0,12 \\
\hline Sulfo G & 3,5 & 1,5 & 1,5 \\
\hline Rhodamine B & 17 & 2,3 & 4,2 \\
\hline
\end{tabular}

${ }^{*}$ ) Après linéanisation à la concentration d'injection

25/ Corrélation statique-dynamique avec le sable de l'Isère.

Dans tous les cas, on a linéarisé l'isotherme en reliant l'origine au point d'équilibre correspondant à la concentration d'injection. comme le montre la figure 23 .

Les premiers résultats dénotent une corrélation satisfaisante entre ces valeurs de $(m)$ obtenues par les essais statiques et dynamiques.

\section{Conclusions}

\subsection{COMMENTAIRES SUR LE MODELE UTILISE}

8.1.1. Facilités offertes par l'utilisation de ce modèle

Le modèle M.C.E., auquel nous avons eu recours pour l'interprétation sommaire de nos essais, avait été jusqu'ici adopté par les seuls chromatographes. Ses notations, peu familières aux hydrologues, peuvent être aisément transposées par eux grâce à l'équivalence formelle avec le modèle P.D.E. dont la signification des paramètres est plus intuitive. L'intérêt essentiel de ce modèle nous parait être sa facilité d'emploi, grâce aux métho- 
des d'ajustement par des critères de forme ne nécessitant, n'en déplaise aux informaticiens, pas d'autre instrument qu'un double décimètre, rusticité de traitement qui ne peut que séduire les praticiens de terrain.

\subsubsection{Inconvénients du modèle}

Ce modèle ne permet de traiter en toute rigueur que les cas simples de sorption linéaire et réversible et de cinétique d'échange du premier ordre, conditions rarement rencontrées en fait dans les processus étudiés.

\subsubsection{Simplification du modele et remarque sur son inter-} prétation hydrologique

Les ajustements effectués sur les réponses impulsionnelles de laboratoire et de terrain confirment une observation de Villermaux (1975). Il a signalé la valeur élevée du paramètre $(J)$, c'est-à-dire, dans la transposition au modèle P.D.E., une valeur élevée du nombre de Peclet, laquelle implique une dispersion (au sens cinématique) faible.

Dans la plupart des processus de migration, la valeur élevée de $(P)$, assimilable à $(P \infty)$ permet de réduire le modèle M.C.E. aux deux paramètres $(N)$ et $(R)$.

Dans le cas précis du transfert du fluide vecteur, si on utilise un bon traceur de l'eau conduisant à des valeurs très proches de $R=1$, ne subsiste plus qu'un seul paramètre $(N)$, caractéristique de la cinétique d'échange et non pas de la dispersion apparente.

Cette analyse doit inciter au réexamen de l'approche suivie jusqu'ici par les hydrodynamiciens et de toutes façons à une révision de la terminologie usitée jusqu'ici.

\subsection{REGLES PRATIQUES ET DIRECTIONS DE RECHER- CHE POUR UNE APPROCHE PLUS FINE DES PHENO- MENES}

8.2.1. Règles pratiques pour une approche sommaire des processus de sorption (figure 26).

La démarche la plus rationnelle pour appréhender les processus de migration en zone saturée consiste à procéder d'abord en laboratoire à des essais statiques. Cinétiques et isothermes de sorption permettent de juger de la validité du modèle M.C.E. Dans ce cas favorable, on dispose des paramètres $(m)$ pente de l'isotherme et $(k)$ constante de vitesse de réaction. On procède ensuite à un essai sur colonne qui fournit la valeur du paramètre $(N)$, caractéristique de la cinétique d'échange liée à la porosité du matériau reconstitué, et de $(R)$ lié à l'équilibre de l'échange.

C'est à ce niveau, en laboratoire, que l'on procède à l'étude comparative du comportement des différentes substances, étude effectuée sur un échantillon moyen représentatif du terrain concerné.

Les essais en vraie grandeur sur site représentatif, qui permettent de transposer les résultats expérimentaux de percolation sur colonne aux transferts réels dans lesnappes aquifères, peuvent être limités à deux substances dont on "décodera" les D.T.S. afin de distinguer les paramètres hydrodynamiques de convection - dispersion des paramètres d'interaction. Cette opération permet de juger de la correction à apporter aux paramètres $(N)$, et éventuellement $(R)$, obtenus en laboratoire dans des conditions de porosité et de vélocité évidemment bien différentes.

En fait, les difficultés expérimentales d'obtention des isothermes et des cinétiques de sorption inciteront à inverser l'ordre des opérations de laboratoire, les essais statiques pouvant n'être entrepris que dans le cas où les bilans de restitution sur colonne se révèleraient très inférieurs à l'unité, dénotant ainsi une sorption non réversible. L'analyse paramétrique comparée de cette D.T.S. avec la D.T.S. d'une substance de référence obtenue conjointement devrait permettre en outre de dépister une cinétique plus complexe.

\subsubsection{Remarque sur la relation entre coefficient d'échange $(N)$ et la constante de vitesse $(k)$}

On ne peut assurer que la constante de vitesse qui figure dans l'expression du coefficient d'échange $(N)$ et celle obtenue par des essais statiques soient identiques. L'interprétation des expressions développées de ces coefficients proposées par Glueckauf (statique) et Villermaux (dynamique) laissent à penser que le coefficient $(k)$, en essais dynamiques, varierait de façon monotone avec la racine carrée de la vitesse intersti-

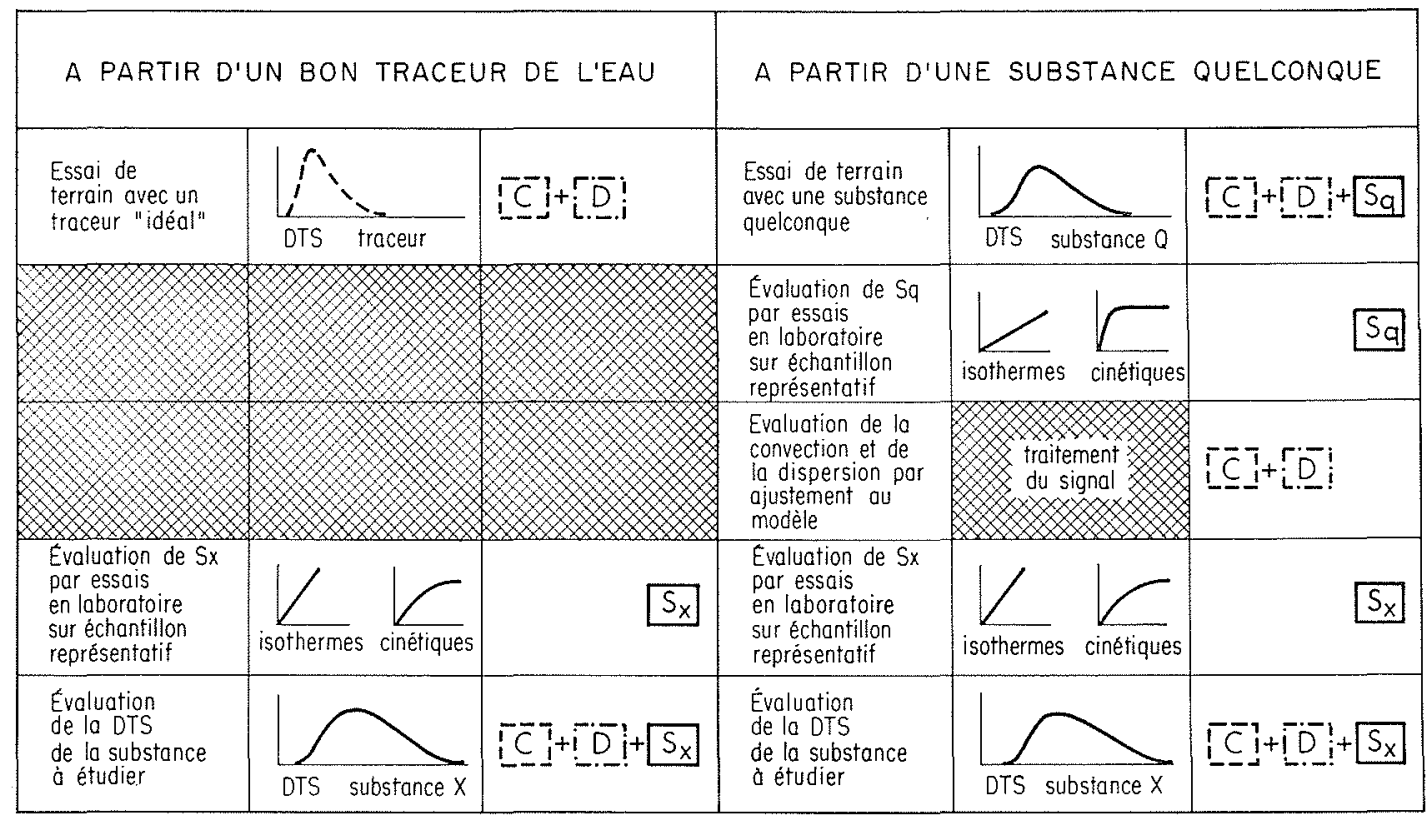

26/ Evaluation des modalités de transport d'une substance miscible 
cielle $(\sqrt{u})$. Cette hypothèse doit être vérifiée si l'on souhaite tirer le meilleur parti des essais dynamiques de laboratoire.

\subsubsection{Approche plus fine des phénomènes}

Comme on l'a mis en évidence sur les figures 18 et 19, l'impossibilité de "linéariser" l'isotherme de sorption (souvent envisageable dans la mesure où les gammes de concentration rencontrées dans des processus de propagation réels sont peu étendues) conduirait à des modèles plus complexes que le modèle M.C.E.

La non réversibilité contraindrait à recourir à des modèles de type Gayraud (1974) excluant toute autre évaluation que numérique (Rochon-Molinari 1974).

\subsection{PERSPECTIVES SUR LES DEVELOPPEMENTS DE LA METHODE}

Après le panorama que nous nous sommes efforcés de rendre exhaustif sur les différentes approches du problème du transport de l'eau et des substances en solution en zone saturée, il nous parait utile de rappeler qu'à l'heure actuelle, la plupart des modèles de propagation mis en oeuvre en milieu saturé ne prennent en considération que la vitesse moyenne de transit de l'eau, impliquant en cela un modèle "piston non dispersif" à zéro paramètre.

Les modèles actuellement les plus sophistiqués, en invoquant de façon sans doute impropre (comme on l'a signalé en 8.1.3.) le terme de "coefficient de dispersion", font appel à un modèle à un seul paramètre : Le modèle "piston dispersif" (P D).

On conçoit combien le fossé qui sépare les praticiens des chercheurs serait long à combler si l'on s'appliquait à tenir compte dorénavant avec rigueur de tous les processus de sorption indiqués.

Une telle attitude nous paraitrait d'autant plus vaine que deux raisons s'opposent à la sophistication des modèles.

La première réside dans le fait que l'hypothèse de réversibilité des processus de sorption, en péjorant les risques de nuisances, va dans le sens d'un accroissement de sécurité pour tous les problèmes de propagation de pollutions.

La seconde raison est qu'il serait vain de faire intervenir des processus de second ordre dans la modélisation de l'interaction lorsque l'évaluation du terme convectif reste fort approchée, approximation dont on se convainc aisément lorsque l'on considère l'insuffisance, la précarité et le coût d'obtention des données expérimentales servant à ajuster ces données, ainsi que l'énorme complexité des réalités de terrain.

\section{Bibliographie}

AIZEN (A.M.), FlDOTKIN (I.M.), MARUTOVSKH (R.M.) - An anabytical solution for adsorption dynamics taking into account longitudinal diffusion -- Russian J. Phys. Chem., 47, pp. 1015-1017, 1973.

ARIS ( $R$ ) The Tongitudinal diffusion coefficient in flow through a tube with stagnant pocketts - Chem. Eng. Sci., 11, pp. 194-198, j959.

CASTANY (G.) -... Traité pratique des eaux souterraines, Dunod Paris, 1963,657 pages.

DE CAZENOVE (E.) - Variation de la perméabilité moyenne des allu. vions hétérogènes en fonction de l'épaisseur noyée - Colloque $\mathrm{In}$ ternational sur les eaux souterraines, Décembre 1970, Palermo.

CLEARY (R.W.), ADRIAN (D.D.) - Analytical solution of the convective dipersive equation for cation adsorption in soils - Soll. Sci. Soc Amer. Proc., 37 (2), pp. 197-199, 1973.
COATS (K.H.), SMiTH (B.D.) -.. Dead-end pore volume and dispersion in porous media - Soc. of Petr. ling. J., Vol 4. pp. 73-84, 1963.

DAVIDSON (J.M.), CHANG (R.K.) - Transport of Picloram in relation to soil physical conditions and pore-water velocity - Soll. Sci. Soc: Amer. Proc., 36, pp. 257-261, 1972.

DEANS (H.A.) - A mathenatical model for dispersion in the direction of flow in porous media - Trans. AI.M.E., Vol 228, $\mathrm{n}^{\circ} 49,1963$

FRIED (J.J.) - Etude théorique et méthodologique de la dispersion en milieu poreux naturel. Thèse Docteur es-sciences - Universite de Bordeaux, Janvier 1972.

GAUDET (J.P.) - Ecoulement transitoire d'eau et de sel dans la zone non saturée. Thèse 3 ème cycle mécanique des fluides, Février 1974 Université Scientifique et Médicale de Grenoble. 136 pages.

GAYRAUD (J.P.) - Etude à l'aide du tritium, de l'iode 131 et du strontium 90 de la dispersion et des échanges physico-chimiques en milieu poreux saturé. Thèse de Docteur de Spécialité, Académie de Montpellier. Université des Sciences et Techniques du Languedoc Décembre 1973,79 pages.

GILES (C.H.), MACEVAN (T.H.), NAKHWA (S.N.), SMITH (D.) Studies in Adsorption, Part XI - J. Chem. Soc. pp. 3973-3991, 1960.

GLUECKAUF (E.) - Ion Exchange and its Applications - Society of Chemical Industries, London, p. 34, 1955.

GUPTA (S.P.), GREEKORN (R.A.) - Dispersion during flow in porous media with bilinear adsorption - Water Resources Research, 9 (5), pp. 1357-1368, 1973.

HANSHAW (B.B.), COPLEN (T.B.) - Ultrafiltration by a compacted clay membrane-Geochimica et Cosmochimica Acta, 37, pp. 22952327,1973

HASHIMOTO (J.), DESHPANDE (K.B.), THOMAS (H.C.) Peclet numbers and retardation factors for ion exchangecolumns - Ind. Eng. Chem. Fund.,3, pp. 213-218; 1964

HORNSBY (A.G.), DAVIDSON (J.M.) -- Solution and adsorbed fluometuron concentration distribution in a water-saturated soil : experimental and predicted evaluation --Soil. Sci. Amer. Proc., 37, pp. $823-828,1973$.

HOUGHTON (G.) - Band shapes in non-linear chromatography with axial dispersion - J. Phys, Chem. Vol 67, pp. 84-88, 1963

HOUGHXON (G.) - The additivity of rate and diffusion phenomenal in continuous chromatography $-J$. of Chromatography, $15, \mathrm{pp}$. $5-8,1964$.

KAY (B.D.), ELRICK (D.E.) - Adsorption and movement of lindane in soils - Soil. Sci., 104, 314-322, 1967.

KYTE (W.S.) - Non linear adsorption in fixed beds : the Freundlich isotherm - Chem. Eng. Sci. Vol 28, pp. 1853-1856, 1973.

LAI SUNGHO, JURINAK (J.J.) - Cation absorption in one-dimensional flow through soils : a numerical solution-Water Resources Research., 8(1), pp. 99-107, 1972.

LALLEMAND (A.) - - Essais en laboratoire de douze traceurs radio actifs, Modalité de leur utilisation pour des études hydrogéologiques -.. Rapport BRGM - 685 Gl 171 EGA, Octobre 1968 , 123 pages.

LAPIDUS (L.), AMUNDSON (N.R.) - Mathematics of adsorption in beds VI. The effect of longitudinal diffusion in ion exchange and chromatographic columns $-J$. Phys. Chem., 56, pp. 984-988, 1952.

LEES (F.P.) - The determination of the moments of the impulse reponse of chemical processes from the basic transformed equations. Chem. Eng. Sci., 24, pp. 1607-1613, 1969.

LINDSTROM (F.T.), BOERSMA (L.) - Theory of chemical transport with simultaneous sorption in a water saturated porous medium. Soil. Sci, Vol. $110, \mathrm{n}^{\circ} 1$, pp. 1-9, 1970.

LINDSTROM (F.T.), BOERSMA (L.), STOCKARD (D.) - A theory on the mass transport of previously distributed chemicais in a water saturated sorbing, porous medium : isothermal cases-Soil., Sci. 112 pp. 291-300, 1971.

MARGAT (J.), CASTANY (G.) - Dictionnaire français d'hydrogéologie (sous presse) - B.R.G.M.Rapport 74 SGN 253 AME, Juillet 1974 $190 \mathrm{p}$.

MOLINARI (J.) -- Interventions au Xième Congrès International de l'A.I.H. Montpellier 16-22 Septembre 1974-Memoires tomeX-2, BRGM, Paris 1975, 344 pages. 
MOLINARI (J.), PEAUDECERF - Etude méthodologique des caractéristiques de transfert des substances chimiques dans les nappes ATP Hydrogeologie - Comptes rendus des Colloques Dispersion des fluides miscibles en milieux poreux et IHydrogéologie des Cal caires fissurés - CNRS, Avril 1975, Institut de Mécanique Grenoble.

ODDSON (J.K.), LETEY (J.) WEEKS (L.V.) - Predicted distribution of organic chemicals in solution and adsorbed as a function of position and time for various chemical in soil proporties -- Soil Sci. Soc. Amer, Proc., 34, pp. 412-417, 1970.

OGATA, - Mathematics of dispersion with linear adsorption isotherm. U.S. Geological Survey Professional Paper, 411-H, 1964, 7 pages

OzıL (P.) - Adsorption dynamique sur tamis moléculaire en lit fixé Thèse de spécialité en chimie minérale, Juillet 1974 Université Scientifique et Médicale de Grenoble, 124 pages.

PFANNKUCH (H.O.) - Contribution à l'étude des déplacements de fluides miscibles dans un milieu poreux. Thèse de Docteur-Ingénieur,

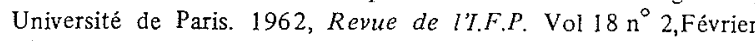
1963, pp. 215-270.

PREUL (H.C.), KHERA (A.K.) - Travel of polluants through an aquifer Eng. Bull. Purdue Univ., 5, pp. 1023-1042, 1971.

ROCHON (J.) - Etude bibliographique sur les mécanismes de sorption en milieu aqueux - Rapport CEA-CENG-SARR/74-12, 1974, 48 pages.

ROCHON (J.), MOLINARI (J.) - Etablissement d'équations de bilan et définition des principaux paramètres caractérisant les processus hydrodynamiques et physico-chimiques de transfert en milieu poreux -- Rapport CEA-CENG - SARR/74-34, 1974, 49 pages.
TAGAMETS (T.), STERNBERG (Y.M.) - A predictor-corrector method for solving the convection-dispersion equation for adsorption in porous media-Departement of Civil Engeneering, University of Maryland. College Park, Maryland 20742, 1974.

VAN GENUCHTEN (M.Th.), DAVIDSON (J.M.), WIERENGA (P.J.) An evaluation of kinetic and equilibrium equations for the prediction of pesticide movement through porous media - Soil. Sci. Soc Amer, Proc., Vol 37, pp. 29-35, 1974.

VILLERMAUX (J.) - Vème Symposium International sur les méthodes de séparation : Chromatographie sur colonne. Lausanne 1969 Chimia - Suppl. 1970, pp. 66-85.

VILLERMAUX (J.) - Analyse des processus chromatographiques linéaires à l'aide de modèles phénoménologiques - Chem. Eng. Sci., vol 27, pp. 1231-1243, 1972

VILLERMAUX (J.) - Relations entre la forme des pics chromatographiques et les paramètres physiques et opératoires de la colonne. Journal of Chromatography, 83, pp. 205-222, 1973.

VILLERMAUX (J.) - Intervention à une réunion du groupe "Dispersion et Echanges en milieux poreux" Antibes (Juin 1975).

WARNCKE (D.D.), BARBER (S.A.) - Diffusion of zinc in soils : III, Relation to zinc adsorption isotherm --Soil. Sci. Soc. Amer. Proc. vol. 37 , pp. $355-358,1973$.

WIERENGA (P.J.), VAN GENUCHTEN (M.Th.) - Solute movement in sorbing porous media with lateral diffusion - Compte rendu des Colloque $A T P$ hydrogeologie du CNRS. Edité par l'Institut de mécanique. Université Scientifique et Médicale de Grenoble. Sept. 1975,13 pages.

\section{Discussion}

Président : M. Jean MA R GAT

M. Le Président remercie M. MOLIN AR I de son exposé très suggestif et ouvre la discusison :

"Vous semblez penser, dit M. BONNET (B.R.G.M.), que les hydrodynamiciens ne sont pas conscients de l'intervention simultanée des phénomènes de convection, dispersion et adsorption dans la propagation en milieux poreux. En fait, la convection a un rôle certainement non négligeable dans les problèmes réels en raison de l'hétérogénéité en perméabilité du terrain. Il faut donc mettre en jeu des modèles réalistes vis-à-vis de la convection, c'est-à-dire des modèles qui tiennent compte de toutes ces hétérogénéités. On s'aperçoit alors qu'il est très difficile de prendre en compte en même temps la diffusion.

"Pour des raisons essentiellement numériques, on n'arrive pas à se débarrasser d'une dispersion numérique qui peut être aussi (ou même parfois plus) importante que la dispersion réelle. Le terme d'adsorption pose moins de problèmes.

"En définitive, les hydrodynamiciens pensent qu'il faudrait résoudre la première difficulté avant d'aborder l'influence de phénomènes réels mais secondaires par rapport à la convection".

M. de MARsily estime qu'il s'agit d'une question d'échelle. Pour l'ensemble d'une nappe, la zone de mélange devenant très vite importante, la dispersion cinématique ne peut être un phénomène secondaire. Cela devient possible dans certains cas à petite échelle (celle à laquelle a travaillé M. MOLINARI).

"On peut, poursuit $M$. de MARsily, montrer, en étudiant l'équation complète du mouvement en milieu poreux, tenant compte de l'adsorption, que vous avez utilisée, que :

- si l'isotherme d'adsorption est linéaire,

- et la cinétique d'adsorption instantanée

alors l'influence de l'adsorption se traduit par une modification apparente de la vitesse de pore, et non du coefficient de dispersion.

"Je me demande alors si les faibles coefficients de dispersion que vous semblez obtenir sont dûs

- à la cinétique d'adsorption, ou à la non linéarité des isothermes (d'ailleurs très visible sur les graphiques" log-log" que vous avez montrés).

"Je crois plutôt que, dans les milieux poreux réels, la dispersion cinématique est importante et la cinétique d'adsorption relativement proche de "l'instantanée" (vu la faible vitesse des circulations). On doit peut être pouvoir interpréter vos expériences de cette façon, et non avec une cinétique élevée et une dispersion faible.

\section{J. ROCHON (C.E.N.G./S.A.R.R.) intervient en ces termes}

"Le modèle présenté tient compte de la convection-dispersion. M MOLINARI a voulu souligner que, lorsque des courbes de restitution présentent, comme celles que nous avons montrées, une dissymétrie très marquée autour de leur valeur modale, la forme de la réponse impulsionnelle est plus affectée par les phénomènes d'echange responsables de la "trainée" des courbes que par la dispersion cinématique qui, dans ces modèles, régit la forme du pic. Dans le type d'ajustement que nous avons adopté, une forme de pic très effilée, telle que nous l'observons généralement, est caractéristique de nombres de Péclet élevés, et par conséquent de dispersions faibles.

"Nous cherchons actuellement à montrer de quelle façon le paramètre $\mathrm{N}$, caractéristique de l'échange, varie avec la vitesse intersticielle".

A l'heure actuelle, la question reste en suspens, toutefois, nos points de vue se concilient sans doute en partie, en tenant compte du fait que, si, dans le milieu poreux réel, la cinétique d'adsorption peut être négligée en raison de temps de séjour élevés, en milieu reconstitué sur colonne, la cinétique doit être davantage prise en considération. Tout ceci fait malheureusement abstraction de la morphologie qui, chaque fois que nous y avons accès, révèle, comme le soulignait $\mathrm{M}$. BONNET, une hétérogénéité extrême dont l'incidence sur les processus de transfert est indéniable et qui est, faute de mieux, globalement prise en compte par les paramètres arbitraires que nous sommes contraints de définir, conclut M. MOLINARI.

M. Le Président clôt la discussion et donne la parole successivement à M GAUDET et à M. JEGAT pour l'exposé de la communication qu'ils ont établie en collaboration avec M.G. VACHA UD. 